\title{
Cockroaches adjust body and appendages to traverse cluttered large obstacles
}

\author{
Yaqing Wang, Ratan Othayoth, *Chen Li \\ Department of Mechanical Engineering, Johns Hopkins University \\ *Corresponding author. Email: $\underline{\text { chen.li@jhu.edu }}$
}

Keywords: terrestrial locomotion, locomotor transitions, potential energy barrier, terradynamics, kinematics

\begin{abstract}
To traverse complex natural terrain, animals often transition between locomotor modes. It is well known that locomotor transitions can be induced by switching in neural control circuits or be driven by a need to minimize metabolic energetic cost. Recent work discovered that locomotor transitions in complex 3-D terrain cluttered with large obstacles can also emerge from physical interaction with the environment controlled by the nervous system. To traverse cluttered, stiff grass-like beams, the discoid cockroach often transitions from using a strenuous pitch mode to push across to using a less strenuous roll mode to maneuver through the gaps, during which a potential energy barrier must be overcome. Although previous robotic physical modeling demonstrated that kinetic energy fluctuation from body oscillation generated by leg propulsion can help overcome the barrier and facilitate this transition, the animal was observed to transition even when the barrier still exceeds kinetic energy fluctuation. Here, we further studied whether and how the cockroach makes active adjustments to facilitate this locomotor transition to traverse cluttered beams. We observed that the animal flexed its head and abdomen, reduced hind leg sprawl, and used both hind legs differentially during the pitch-to-roll transition, which were absent when running on a flat ground. Using a refined potential energy landscape with additional degrees of freedom modeling these adjustments, we found that head flexion did not substantially reduce the transition barrier, whereas the leg sprawl reduction did so dramatically. We discussed likely functions of the observed adjustments and suggested future directions.
\end{abstract}




\section{Introduction}

Animals locomotion emerges from direct physical interaction with the environment controlled by the nervous system, via both feedforward preflexes facilitated by morphology and feedback control modulated by sensing (Dickinson, 2000). To move across complex environments, animals often use and transition between multiple modes of locomotion (Alexander, 2006; Dickinson, 2000; Li et al., 2015; Lock et al., 2013; Low et al., 2015). Most terrestrial locomotion studies focused on how animals use

34 neuromechanical control to generate or stabilize near-steady-state, single-mode locomotion (e.g., walking, running, (Blickhan and Full, 1993; Kuo, 2007)). Previous work explored how gait transitions result from changes in the rhythmic output of central pattern generators (Ijspeert, 2008), sensed information of the environment (Blaesing and Cruse, 2004; Ritzmann et al., 2012), or the need to minimize energetic cost over large spatiotemporal scales (Bramble and Lieberman, 2004; Shepard et al., 2013). Only very recently have we begun to understand that in complex 3-D terrain locomotor transitions can emerge from animals' direct physical interaction with the environment (Othayoth et al., 2020; Othayoth et al., 2021).

Our study was motivated by and builds upon this recent study, which concerns the discoid cockroach (Blaberus discoidalis) traversing a layer of cluttered grass-like beam obstacles (Othayoth et al.,

44 pitching body (the pitch mode), but then it often rolls its body into a gap between beams (the roll mode) to state is attracted to local minimum basins of an underlying potential energy landscape. Traversing stiff beams using the pitch mode is more strenuous than using the roll mode because it requires overcoming a system transitions from the pitch to the roll mode when the kinetic energy fluctuation from body oscillations

50 (which result from cyclic leg propulsion) exceed a potential energy barrier between the pitch and roll basins.

51 However, despite qualitatively similar overall findings, the animal's pitch-to-roll transition happens even 

must also be making active adjustments to facilitate the transition.

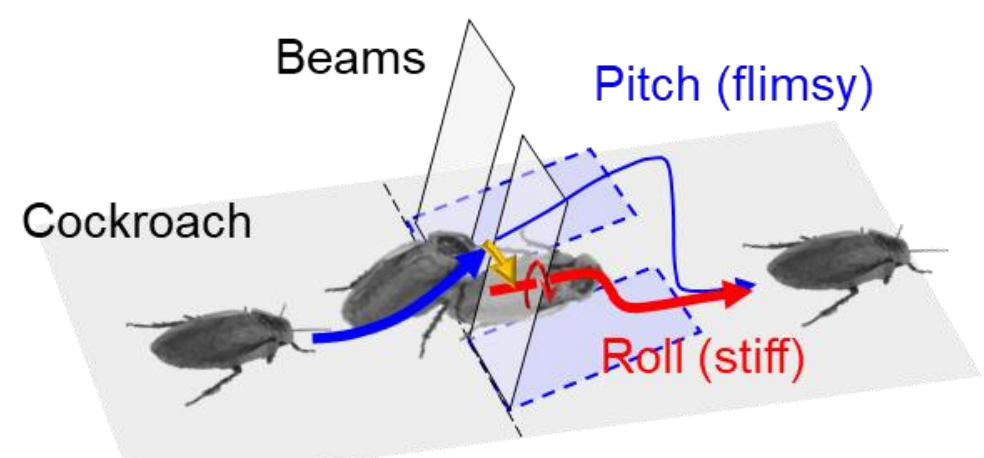

Fig. 1. Cockroach transitioned from pitch to roll mode to traverse beams. Adapted from (Othayoth et al., 2020).

Here we take the next step to further explore how the discoid cockroach make adjustments to facilitate pitch-to-roll transition. We challenged the animal to traverse a layer of stiff beams and used high speed imaging to measure detailed body and appendage kinematics. We discovered that the animal makes several adjustments: (1) Head flexion: the animal flexed its head up and down multiple times while interacting with the beams. (2) Abdomen flexion: the animal flexed its abdomen after the animal rolled into the beam gap. (3) Leg sprawl reduction: the animal spread its hind legs outward when pitching against the beams, but it tucked its hind legs inward when rolling into the beam gap. (4) Differential leg use: the animal extended one hind leg while retracting the other when rolling into the beam gap. animal transitioned from the pitch to the roll mode, active head flexion reduces the transition barrier and facilitates rolling into the gap. (2) When the animal body long has rolled into the gap, active head flexion facilitates it staying within the gap. We also hypothesized that the animal actively adjusts hind legs sprawl to facilitate pitch-to-roll transition. Specifically: (3) When pitching up against the beams, a large leg sprawl sprawl reduces the pitch-to-roll transition barrier. 

and how much the observed use of head flexion and leg sprawl reduction changed the potential energy

74 barrier that must be overcome to transition from the pitch to the roll mode (which measures the difficulty

75 of the transition). To test hypotheses (2), we analyzed whether and how much the observed use of head

76 flexion changed the potential energy barrier that to transition from the rolled body being within the gap

77 between the beams to being out of the gap and deflected sideways. We found that only leg sprawl reduction

78 reduced the pitch-to-roll transition barrier. Finally, we discuss the likely functions of the observed body and 79 appendage adjustments and suggest future directions.

\section{Methods}

82

\subsection{Animals}

We used three male Blaberus discoidalis cockroaches (Joe's BUGz LLC, Atlanta, GA, USA). Before the experiments, each animal was kept in a plastic container in a room with a controlled temperature of $22^{\circ} \mathrm{C}$, moisture of $70 \%$, and lighting on a $12 \mathrm{~h}: 12 \mathrm{~h}$ light-dark cycle. Dry dog food (Purina Beneful, Largo, FL, USA) and water jelly made from water and polymer crystal (Tasty Worms Nutrition Inc., USA) were provided ad libitum. The animals weighed $2.7 \pm 0.6 \mathrm{~g}$ (with marker items) and measured $5.3 \pm 0.3 \mathrm{~cm}$ in length, $2.3 \pm 0.1 \mathrm{~cm}$ in width, and $0.73 \pm 0.08 \mathrm{~cm}$ in thickness. All data reported are means \pm s.d. unless specified otherwise.

\subsection{Obstacle track}

For controlled, repeatable experiments, we constructed a testbed (Fig. 2A) similar to that in the previous study (Othayoth et al., 2020), with a layer of beam obstacles that consisted of seven beams. Each beam was $10 \mathrm{~mm}$ wide, $100 \mathrm{~mm}$ tall, and $0.8 \mathrm{~mm}$ thick. The lateral distance between two adjacent beams was $10 \mathrm{~mm}$, and the lateral distance between the left/right-most beams and the walls was $5 \mathrm{~mm}$. We used the same method to construct beam obstacles and characterize their stiffness as described in the previous 


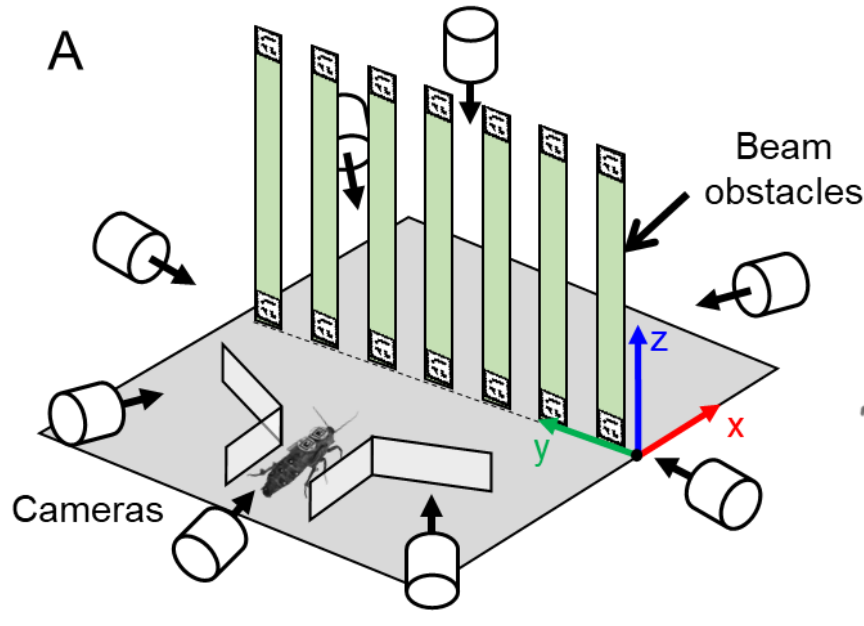
probability (Othayoth et al., 2020) to increase experimental yield.

\subsection{Imaging setup} the ambient temperature around the arena was around $41^{\circ} \mathrm{C}$.

study (Othayoth et al., 2020). The beams can only deflect about a hinge just above the ground. The beam torsional stiffness was $K=2.5 \pm 0.4 \mathrm{mN} \cdot \mathrm{m} / \mathrm{rad}$ (mean \pm s.d. of 7 loading cycles), between the two most stiff beams in the previous study. We chose this high stiffness to induce a high pitch-to-roll transition

Eight synchronized high-speed cameras (N5A-100, Adimec, Netherlands) recorded the experiment from different views: one from dorsal view, two from the side view, one from the oblique top view, and four from isometric views (Fig. 2A). All the cameras recorded at a frame rate of $100 \mathrm{~Hz}$, a shutter time of $50 \mu \mathrm{s}$, and a resolution of $2592 \times 2048$ pixels. Even with eight cameras, we had to carefully tune camera positioning and orientation to achieve reliable tracking of the animal and beams (Sec. 2.5), because the animal had large 3-D body rotations ( $\max$ absolute body yaw $=100^{\circ}$, max absolute pitch $=62^{\circ}$, $\max$ absolute roll $=98^{\circ}$, defined in Sec. 2.6) and markers were frequently occluded by the beams. Four work lamps (Coleman Cable, Waukegan, IL, USA) provided lighting from the top and side. During experiments,

111 Fig. 2. Experimental setup. (A) Schematic of beam obstacle track and multi-camera imaging system. (B)

112 Marker placement on animal and definition of thorax frame. Magenta: head marker. Yellow: thorax marker.

113 Cyan: abdomen markers. Orange: leg markers. See body frame definition in Sec. 2.6. 


\subsection{Experiment protocol}

During each trial, we first placed the animal at the start of the track, covered it with a piece of

116 cardboard, and let it settle down. We recorded the ambient temperature around the obstacle field and reset

117 the beams to an upright pose. Then we started camera recording, lifted the cardboard to expose the animal

118 to bright light, and prodded its abdomen with a tape-wrapped straw to induce running through the funnel

119 towards the beams. After the animal traversed the beams, it entered a shelter of egg cartons (not shown) at

120 the other end of the track. Then, camera recording was stopped, and videos were saved. The animal was

121 allowed at least 3 minutes to rest after each trial. For each animal, we recorded 18-19 trials.

122 For each animal, we rejected the trials in which at least one of the following situations occurred:

123 (1) The animal used at least one locomotor mode (Li et al., 2015) other than the pitch and roll modes

124 (Othayoth et al., 2020) to traverse the beams. (2) The animal touched the arena wall in the roll phase

125 (defined in Sec. 2.7). (3) At least one marker (BEEtags, white-outs, or beads) fell off. From the remaining

126 trials, we selected the 12 trials with the least traversal time for each animal, with a total of 36 trials $(N=3$,

$127 n=36)$.

\subsection{Tracking and $3-\mathrm{D}$ reconstruction}

To overcome the challenge in tracking from large 3-D body rotation and frequent occlusions, we

130 used several types of markers (Fig. 2B). (1) We glued the animal's wings into a natural folded shape using

131 hot glue and exposed the abdomen by trimming the posterior half of the wings. Then we used hot glue to

132 attach a BEEtag (Crall et al., 2015) to the anterior half of the fixed wings covering the thorax as the thorax

133 marker (Fig. 2B, yellow). (2) We used hot glue to attach a BEEtag onto the animals' pronotum as the head

134 marker (Fig. 2B, magenta). (3) We used white-out to paint point markers on the dorsal surface of the

135 abdomen as abdomen markers (Fig. 2B, cyan). (4) We used ultraviolet curing glue (Bondic, Aurora,

136 Ontario, Canada) to attach two small, lightweight (12 mg each) aluminum beads (McMaster-Carr, Elmhurst,

137 IL, USA) to each hind leg at two locations close to the femoral-tibial and tibia-tarsal joints as leg markers. 
138 (Fig. 2B, orange) (5) We attached BEEtags (Crall et al., 2015) to the top and the bottom of each beam's

139 frontal side as beam markers (Fig. 2A).

140 Then, we tracked the markers on the animal and beams in each recorded video from all eight

141 cameras. We tracked all the BEEtag markers automatically using a customed MATLAB code modified

142 from the BEEtag code (Crall et al., 2015). To track the abdomen and leg markers efficiently, we used

143 DeepLabCut (Mathis et al., 2018). For each camera view, we first manually digitized these markers in 10

144 trials, with 100 video frames from each camera view, and used these data as a training sample to train the

145 neural network. After training, DeepLabCut tracked the markers in the videos. We then visually examined

146 the sample tracking results, manually fixed obvious tracking errors, and re-trained the training sample. After

147 several cycles of manual corrections and reinforcement learning, the DeepLabCut could automatically track

148 markers with high accuracy. We visually checked the tracking result carefully and manually corrected the

149 remaining tracking errors. Using this tracking method, we achieved a high maker tracking performance: the

150 head, thorax, abdomen, and leg markers were all tracked in $100 \%$ of all the frames of all trials. We

151 emphasize that, even with 8 cameras covering a large angular range and using DeepLabCut, tracking

152 detailed kinematics in such a densely cluttered terrain is a laborious and time-consuming process, due to

153 the large body rotation in 3-D and frequent occlusions of markers. In total, it took an experienced

154 experimenter 20 hours of manual digitizing, 72 hours of automatic tracking, and 150 hours of manual

155 correction to track 36 trials each averaging 280 frames with 8 camera views.

156 Finally, we reconstructed 3-D kinematics of all tracked markers using the Direct Linear

157 Transformation method (DLTcal5) (Hedrick, 2008). To facilitate 3-D calibration, we built a calibration

158 object with 60 BEEtag markers using Lego bricks (The Lego Group, Denmark).

\subsection{Kinematics analysis}

With the 3-D reconstruction of tracked markers, we quantified the motion of the animal's head,

161 thorax, abdomen, and two hind legs. For simplicity, we laterally mirrored kinematic data of the trials in 
162

163

164

165

166

167

168

169

170

171

172

173

174

175

which the animal rolled to the left to become rolling to the right to simplify the analysis, considering lateral symmetry.

We first approximated the thorax frame (which is the body frame in (Othayoth et al., 2020)) and head frame using the BEEtags on them. To do this, we projected a model of the animal (Sec. 2.9) to a set of eight synchronized camera views, and adjusted its pose so that it visually matched the animal figure in the video. We checked this matching between the model and the animal figure in at least 5 other frames in the videos. Then we defined the thorax frame $\left(X_{\mathrm{T}} Y_{\mathrm{T}} Z_{\mathrm{T}}\right)$ and head frame $\left(X_{\mathrm{H}} Y_{\mathrm{H}} Z_{\mathrm{H}}\right)$ as the frame of the model, and used homogeneous transformation between the tags and the models to represent the spatial relationship between the tags and the animal thorax and head parts. For the abdomen frame $\left(X_{\mathrm{A}} Y_{\mathrm{A}} Z_{\mathrm{A}}\right)$, we defined the origin $\left(\mathrm{O}_{\mathrm{A}}\right)$ as the foot of the perpendicular from the top marker $\left(\mathrm{A}_{T}\right)$ to the segment of left $\left(\mathrm{A}_{\mathrm{L}}\right)$ and right markers $\left(A_{R}\right)$, defined the $x$-axis as the direction from the origin $\left(\mathrm{O}_{\mathrm{A}}\right)$ pointing at the top marker $\left(\mathrm{A}_{T}\right)$, defined the $y$-axis as the direction from the origin $\left(\mathrm{O}_{\mathrm{A}}\right)$ pointing at the left marker $\left(\mathrm{A}_{\mathrm{L}}\right)($ Fig. 3A). Thus, we obtained the head, thorax, and abdomen frames, each with 3-D position $(x, y, z)$ and orientation (yaw $\alpha$, pitch $\beta$, roll $\gamma, Z-Y^{\prime}-X^{\prime}$ ' Tait-Bryan convention) (Fig. 3A).

We then calculated the following kinematic variables as a function of time in each trial. (1) Head flexion $\beta_{\mathrm{h}}$, defined as the additive inverse of the pitch of the head frame in the thorax frame. (2) Abdomen flexion $\beta_{\mathrm{a}}$ : the pitch of the abdomen frame in the thorax frame. (3) Leg sprawl $\phi$ : the angle between the two vectors from the thorax frame origin $\left(\mathrm{O}_{B}\right)$ to the leg markers $\left(\mathrm{L}_{\mathrm{L}}, \mathrm{L}_{\mathrm{R}}\right)$ (Fig. 3B). (4) Leg height difference $\Delta h$ : The leg height of the left hind leg $\left(\mathrm{h}_{\mathrm{L}}\right)$ minus the leg height of the right hind leg $\left(\mathrm{h}_{\mathrm{R}}\right)$ (after mirrored). Hind leg height was defined as the distance of the leg marker from the thorax coronal plane (Fig. 3B). All the Equations are summarized below.

The rotation matrix of the thorax $\left(R_{\mathrm{B}}\right)$, head $\left(R_{\mathrm{H}}\right)$, and abdomen $\left(R_{\mathrm{A}}\right)$ frames in the world frame:

$$
R=\left[\begin{array}{ccc}
c_{\alpha} c_{\beta} & \mathrm{c}_{\alpha} \mathrm{s}_{\beta} \mathrm{s}_{\gamma}-\mathrm{s}_{\alpha} \mathrm{c}_{\gamma} & c_{\alpha} s_{\beta} c_{\gamma}+s_{\alpha} s_{\gamma} \\
c_{\alpha} s_{\beta} & \mathrm{c}_{\alpha} \mathrm{s}_{\beta} \mathrm{s}_{\gamma}+\mathrm{s}_{\alpha} \mathrm{c}_{\gamma} & c_{\alpha} s_{\beta} c_{\gamma}-s_{\alpha} s_{\gamma} \\
-s_{\beta} & c_{\beta} s_{\gamma} & c_{\beta} c_{\gamma}
\end{array}\right],
$$


185 Which $s_{\times}$and $c_{\times}$are abbreviations for sine and cosine terms, respectively. $\alpha, \beta$, and $\gamma$ are the Euler angles.

186 The rotation matrix of head and abdomen frames in the thorax frame:

$$
R_{\mathrm{TH}}=R_{\mathrm{T}}^{\mathrm{T}} R_{\mathrm{H}},
$$

$$
R_{\mathrm{TA}}=R_{\mathrm{T}}^{\mathrm{T}} R_{\mathrm{A}},
$$

189 The head flexion and abdomen flexion:

$$
\beta_{\mathrm{h}}=\beta\left(R_{\mathrm{TH}}\right)
$$

$$
\beta_{\mathrm{a}}=-\beta\left(R_{\mathrm{TA}}\right),
$$

192 Where $\beta(\cdot)$ means to obtain the pitch of a rotational matrix:

$$
\beta(R)=\operatorname{atan} 2\left(\sqrt{r_{31}^{2}+r_{32}^{2}}, r_{33}\right),
$$

194 Where $r_{\mathrm{ij}}$ is the $\mathrm{i}^{\text {th }}$ row and $\mathrm{j}^{\text {th }}$ element in the matrix $R$.

195 Leg sprawl:

$$
\phi=\cos ^{-1}\left[\left(\overrightarrow{O_{T} L_{L}} \cdot \overrightarrow{O_{T} L_{R}}\right) /\left(\left\|\overrightarrow{O_{T} L_{L}}\right\| \cdot\left\|\overrightarrow{O_{T} L_{R}}\right\|\right)\right]
$$

197 Leg height difference:

$$
\Delta h=\mathrm{Z}\left(\mathrm{L}_{\mathrm{L}}\right)-\mathrm{Z}\left(\mathrm{L}_{\mathrm{R}}\right),
$$

199 where $\mathrm{Z}(\cdot)$ means to obtain the $z$ coordinate of a point. 

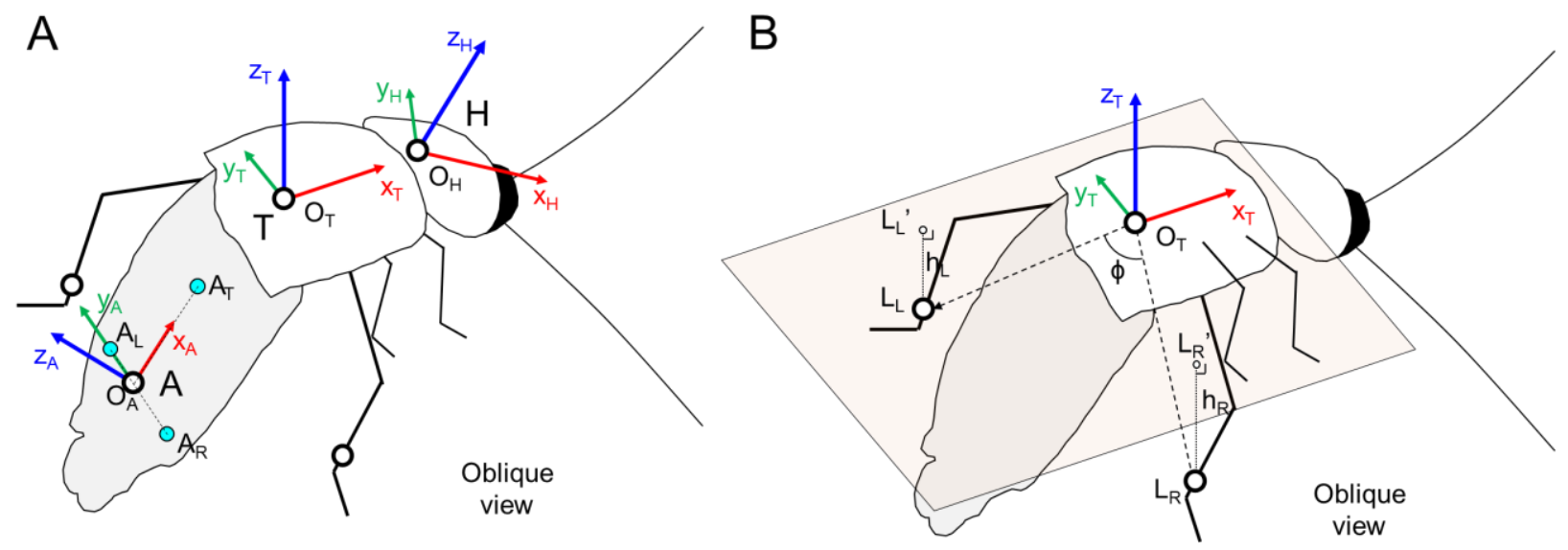

Fig. 3. Schematic diagrams of a cockroach. (A) Coordinate $X_{\mathrm{T}} Y_{\mathrm{T}} Z_{\mathrm{T}}$ : thorax frame. Coordinate $X_{\mathrm{H}} Y_{\mathrm{H}} Z_{\mathrm{H}}$ : head frame. Coordinate $X_{\mathrm{A}} Y_{\mathrm{A}} Z_{\mathrm{A}}$ : abdomen frame. (B) Light-colored plane: thorax coronal plane. $\mathrm{L}_{\mathrm{L}}$ ': Left leg marker. $\mathrm{L}_{\mathrm{R}}$ : Right leg marker. $\phi$ : leg sprawl. $\mathrm{h}_{\mathrm{L}}$ : left leg height. $\mathrm{h}_{\mathrm{R}}$ : right leg height.

\subsection{Definition of traversal phases}

210 distinct phases:

211 (1) Approach (gray): From when the animal ran into the camera view to when it collided with the beams.

212 (2) Explore + Pitch (blue): From when the animal collided with the beams to when it started the final, 213 successful roll attempt. Because the animal sometimes attempted to roll its body more than once, here 214 we separated this phase and the next phase with the start of the last, successful attempt. The start of the 215 attempt was defined as the instance when the animal's body roll changed sign from negative to positive.

216 (3) Roll (red): From when the animal started the final, successful body roll attempt to when body roll was 217 maximal.

218 (4) Land (orange): From when body roll was maximal to when the animal landed with all its six legs had 219 touched the ground again. In all the following analyses in this article, this phase was ignored.

(5) Depart (green): From when the animal landed with all its six legs touching the ground to when it exited the camera view. 
We aligned the time axis of each trial by setting the beginning of the roll phase as $t=0$. For each time instance, we calculated the mean and standard deviation of each variable across all the trials. To compare across traversal phases, we first averaged all kinematic variables (i.e., body roll $\gamma$, body pitch $\beta$, head flexion $\beta_{\mathrm{h}}$, abdomen flexion $\beta_{\mathrm{a}}$, leg sprawl $\phi$, and leg height difference $\Delta h$ ) over time in each phase, animal's repeated head flexion than the average does, we calculated the standard deviation of the head performed using MATLAB (MathWorks, Inc., MA, USA). as a fixed factor, and the individual as a random factor to account for individual variability. These statistical tests were performed using JMP 16 (SAS Institute Inc., NC, USA).

\subsection{Potential energy landscape model definition} mm, width:14.4 mm, thickness: $6.0 \mathrm{~mm}$ ). The abdomen was modeled as half of an ellipsoid (Fig. 4A,

244 yellow, length: $30.5 \mathrm{~mm}$, width: $20.6 \mathrm{~mm}$, thickness: $6.5 \mathrm{~mm}$ ). All the dimensions above were the averages 245 of the measured animal dimensions. When added, the hind legs were modeled as rigid rods with one side

246 fixed to the thorax center (Fig. S2B), because we only tracked the tibia-tarsal joint of the legs. The length

247 of each leg was $27 \mathrm{~mm}$ (average of maximal leg length is $27 \pm 2 \mathrm{~mm}$ in the explore + pitch and roll phases 
over all trials). For simplicity, we assumed that the head and abdomen could each only flex about a lateral (Fig. 4B) to reduce unrealistic concavity between these segments.
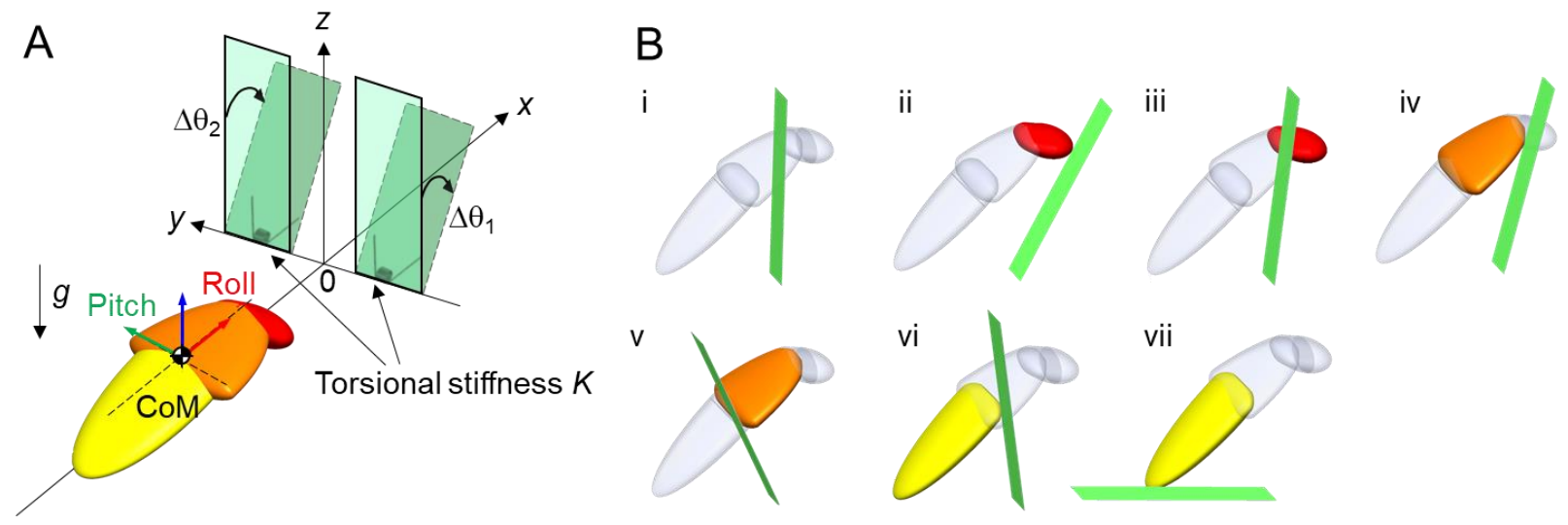

Fig. 4. Modeling for potential energy landscape approach. (A) Model of animal and beams and definition of variables. (B) Schematic examples to choose between multiple possibilities of beam deflection. deflection angle were always selected (Othayoth et al., 2020). This resulted in overestimated beam deflection. In particular, when the cockroach had already traversed the beams using the roll mode, the estimated beam deflections were still calculated as if the beams blocked in front of the cockroach when the animal used a pitch mode. Here, to refine the model, we allowed the beams to deflect either forward or 263 backward during the interaction, and we determined each beam's deflection $\left(\Delta \theta_{1}, \Delta \theta_{2}\right)$ as the angle with minimal absolute value at which the beam did not overlap with any part of the animal. This revised protocol ensured that, when the cockroach is sufficiently far away from the beam, either not having entered the beam area or having already traversed, beam deflection is zero; when the animal is interacting but has not 
backward. Note that this revised protocol does not affect the transition barrier analysis, because the pitch-

to-roll transition happened when the animal body was only beginning to enter the gap(average body $x=$ same result. any body part; in that case, the two possible deflection angles where the beam is tangential to this part are set to be $0^{\circ}$. Then, we rejected the beam deflections where the beam overlaps with any body part (Fig. 4B, i, iii, iv, v, vi), and finally selected the deflection angle with minimum absolute value (Fig. 4B, ii is selected, Fig. 4B, vii is rejected). Beam deflection calculated from this method better matched experimental measurements than in the previous study (Othayoth et al., 2020), reducing the error from $15^{\circ} \pm 32^{\circ}$ to $-1^{\circ}$ $\pm 13^{\circ}(P<0.001$, repeated-measures ANOVA $)$. and beam elastic energy:

$$
E_{\text {potential }}=m_{\text {cockroach }} g z+\frac{1}{2} m_{\text {beam }} g L\left(\cos \Delta \theta_{1}+\cos \Delta \theta_{2}\right)+\frac{1}{2} K\left(\Delta \theta_{1}^{2}+\Delta \theta_{2}^{2}\right),
$$

where $m_{\text {cockroach }}$ is the animal mass, $g$ is gravitational acceleration, $z$ is the body center of mass height from the ground, $m_{\text {beam }}$ is the beam mass, $L$ is the beam length, $K$ is the beam torsional stiffness, and $\Delta \theta_{1}$ and $\Delta \theta_{2}$ are beam deflection from vertical. Given the constraints above, it was fully determined by the animal's position, orientation, and head and abdomen flexion and does not depend on the trajectory (i.e., determined

\section{2.10. Potential energy landscape model generation}


We generated the potential energy landscape in a similar way as in the previous study (Othayoth et al., 2020). The model system has eight degrees of freedom, including the animal position (forward $x$, lateral $y$, vertical $z$ ) and orientation (yaw $\alpha$, pitch $\beta$, roll $\gamma$ ) of thorax and head $\left(\beta_{\mathrm{h}}\right)$ and abdomen $\left(\beta_{\mathrm{a}}\right)$ flexion. So, the potential energy of the system should be a function of these eight independent variables:

$$
E_{\text {potential }}=E_{\text {potential }}\left(x, y, z, \alpha, \beta, \gamma, \beta_{h}, \beta_{a}\right)
$$

We calculated the potential energy landscape over the 8-D space by varying these eight variables, their ranges, and increments are listed in Tab. 1. We did not vary the abdomen flexion $\beta_{\mathrm{a}}$ for two reasons. First, the head and leg adjustments likely facilitated transition to the roll mode, whereas the abdomen which 301 interacted with the beams after the body had already rolled into the gap and likely contributed less to this 302 transition. Second, it is computationally costly to add one more dimension to our potential energy landscape 303 calculations. The first 7 dimensions that we varied systematically already took 3 weeks of computation on 304 a 32-core $2.93 \mathrm{GHz}$ workstation. It would take 20 times more ( a year) if we varied abdomen flexion like 305 head flexion. To simplify landscape analysis, we focused on two cross-sections of the full 8-dimension landscape over a few dimensions of interest by collapsing less relevant dimensions. We first collapsed the landscape along the $z$ dimension. For each combination of the other 7 variables, potential energy is a function of $z$. We varied $z$ from $z_{\min }$ (when the body touched the ground) to $z_{\min }+15 \mathrm{~mm}$ and chose the $z$

309 value for which potential energy is minimal, assuming that the unstable (due to self-propulsion) system was

310 attracted to the minimum. This method was different from that used in the previous study (Othayoth et al.,

311 2020), where the animal's lowest point was constrained to always touch the ground (i.e., ground contract

312 constraint). The body $z$ obtained from this refined method better matched observations: with the ground 313 contact constraint, to reach the average measured $z$ when the animal interacted with the beams $(x=-9$ to

$314-3 \mathrm{~mm}$, Fig. S2B) would require the animal to pitch up by an average of $20^{\circ}$, much greater than the 315 observation $\left(<10^{\circ}\right.$, Fig. S2D) which was only possible without the ground contact constraint. 
function of forward position $x$, we first discretized $x$ within $[-26,33] \mathrm{mm}$ into 296 bins each spanning 0.2

$\mathrm{mm}$. Between each two adjacent time steps in each trial, we checked whether the animal passed any of these

$x$ bins. For each bin where this occurred, we determined the values of kinematic variables other than $x$ using

linear interpolation over $x$ and recorded them under this bin. Finally, we averaged these recorded variables

For the pitch-roll cross-section analysis, we always kept body $y$ and yaw $\alpha$ to follow the average trajectory.

For both analyses, we constrained the abdomen pitch $\beta_{\mathrm{a}}$ fixed at $7^{\circ}$ (temporal average of abdomen flexion

331 To study if the head flexion helped the animal stay within the gap after the animal rolled (Sec. 2.12), we

Table 1. Ranges and increment of landscape variation and dimension collapse protocol. $z_{\min }$ is the $z$

where the lowest point of the body touches the ground.

\begin{tabular}{|c|c|c|c|c|c|c|}
\hline \multirow{2}{*}{ Variable } & \multirow{2}{*}{ Unit } & \multirow{2}{*}{ Min } & \multirow{2}{*}{ Max } & \multirow{2}{*}{ Increment } & \multicolumn{2}{|c|}{ Dimension collapsing protocol } \\
\cline { 4 - 7 } & & & & & Pitch-roll cross-section & Yaw cross-section \\
\hline Forward position $x$ & $\mathrm{~mm}$ & -26 & 33 & 0.2 & NC & NC \\
\hline Lateral position $y$ & $\mathrm{~mm}$ & -3 & 3 & 1 & FAT & FAT \\
\hline Vertical position $z$ & $\mathrm{~mm}$ & $z_{\min }$ & $\begin{array}{c}z_{\min } \\
+15\end{array}$ & 1 & MPE & MPE \\
\hline Yaw $\alpha$ & ${ }^{\circ}$ & -90 & 90 & 5 & FAT & NC \\
\hline Pitch $\beta$ & ${ }^{\circ}$ & -90 & 90 & 2 & NC & FAT \\
\hline Roll $\gamma$ & $\circ$ & -18 & 180 & 2 & NC & FAT \\
\hline Head flexion $\beta_{\mathrm{h}}$ & $\circ$ & -25 & 65 & 5 & NC or FAT & NC \\
\hline
\end{tabular}




\begin{tabular}{|l|l|l|l|l|l|l|}
\hline Abdomen flexion $\beta_{\mathrm{a}}$ & ${ }^{\circ}$ & 7 & 7 & - & - & - \\
\hline
\end{tabular}

336 NC: not collapsed. FAT: follow average trajectory. MPE: minimize potential energy.

337 2.11. Quantifying difficulty of transition using potential energy barrier

First say that you are analyzing the transition barrier from the pitch to the roll mode for hypotheses

To quantify the difficulty to transition between two modes (i.e., from the pitch to the roll mode, from the roll to the deflect mode), we generated the relevant cross-section of the landscape, identified the basins corresponding to these modes, and calculated the transition barrier.

First, we looped through all points on the landscape to identify the local minimum basins (Fig. 5A, ii). 
360 We fed the gridded potential energy landscape cross-section as the graph data, with the starting minimum

361 as the start point, the destination minimum as the goal point, and the highest potential energy on the

362 traversing route as the cost function. The algorithm gave a route from the starting minimum to the

363 destination minimum that crossed the lowest energy barrier (Fig. 5B, C, green curve). We defined the point

364 with the highest energy on this route as the saddle point (Fig. 5B, C, orange point), which was usually a

365 local maximum on a landscape or a true saddle point on a landscape. We defined the transition barrier as

366 the potential energy increasing from the starting minimum to the saddle.

Intuitively, the breadth-first search approach resembled to extrude water at the start point and track

368 the expansion of the water-covering area. The potential energy landscape resembled an uneven surface, and

369 each basin on the surface corresponded with a locomotor mode, such as pitch and roll modes (Othayoth et

370 al., 2020). Enlarging the cost resembled extruding water in the starting basin and increasing the water level.

371 As the water level increased, there was a moment that the water level was high enough to overwhelm a gap

372 between two basins, and the water flowed via this gap into the destination basin. The current water level

373 resembled the potential energy barrier, and the water flow resembled the rest searching sequence in the

374 breadth-first search approach. 
A i

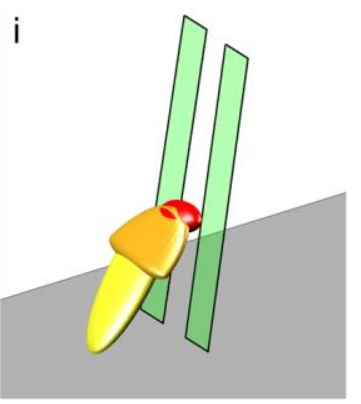

B i

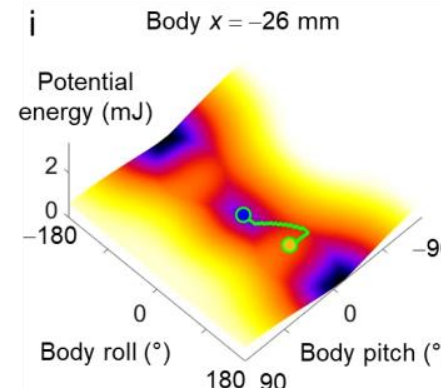

18090

C

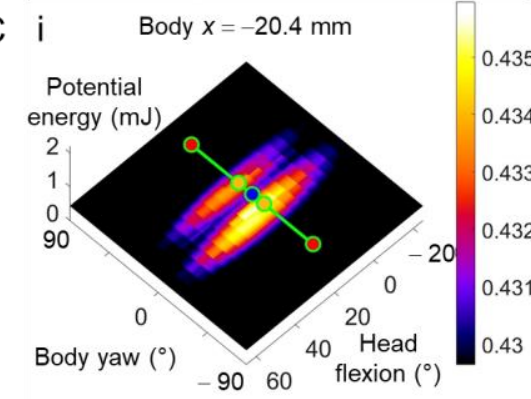

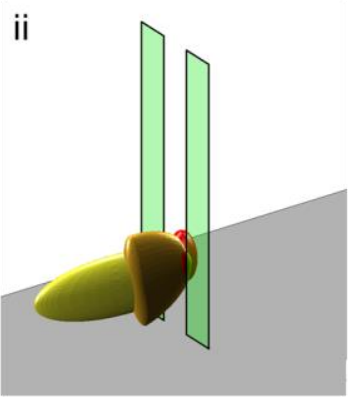
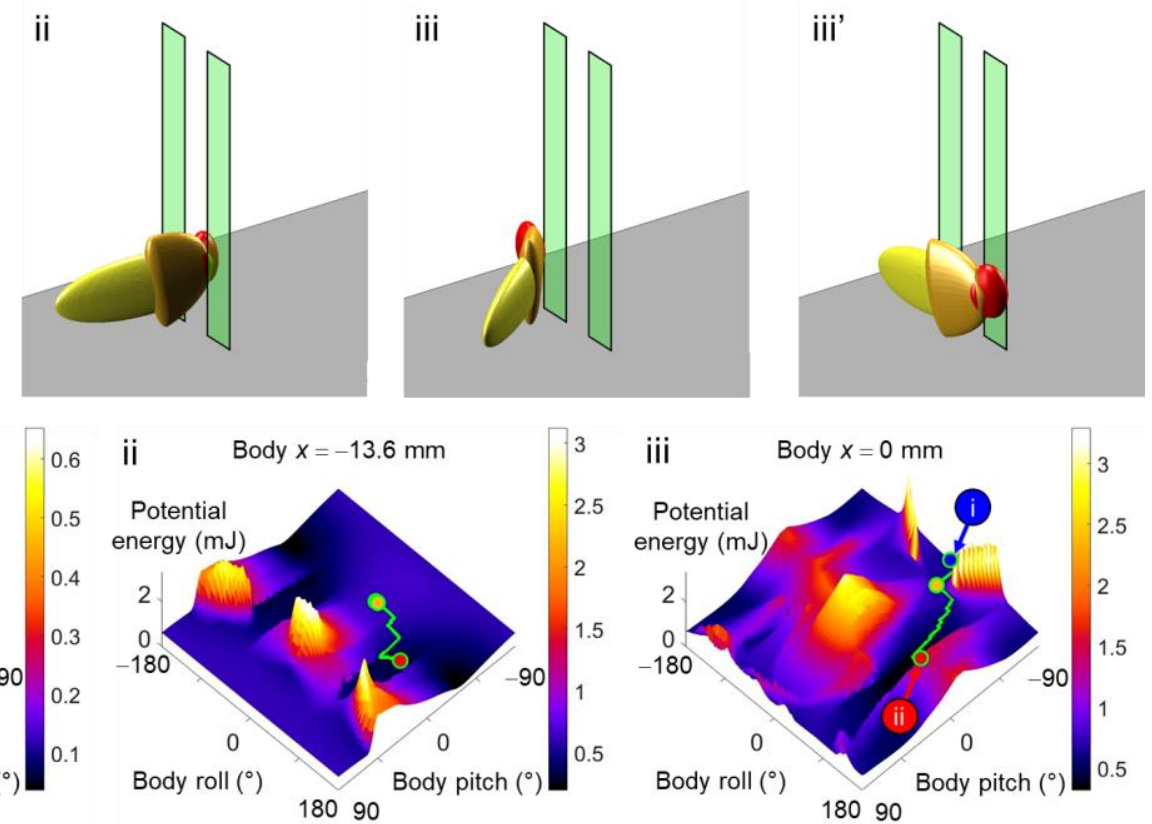

18090

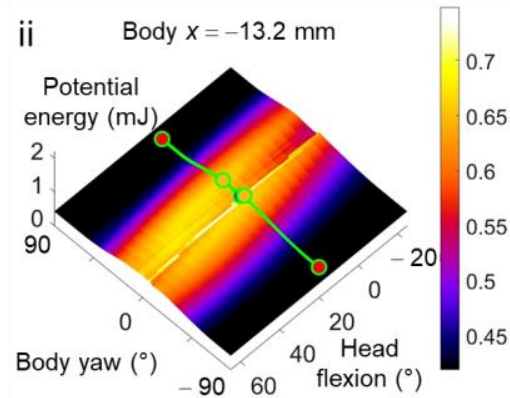

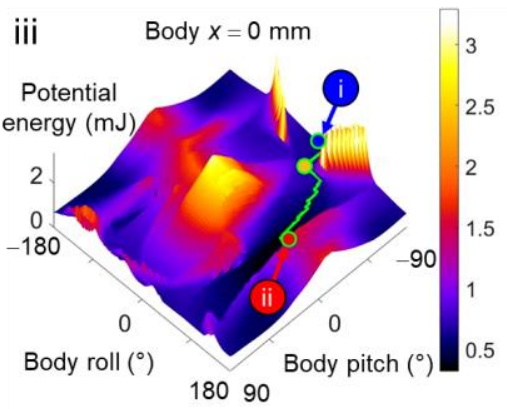

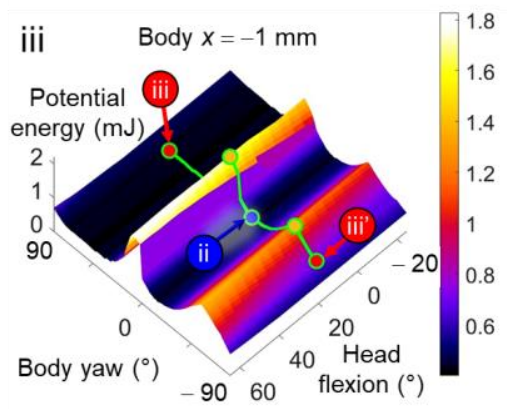

Fig. 5. Evolution of potential energy landscape. (A) Demonstration of locomotor modes. (i) Pitch mode.

(ii) Roll mode, also between-gap mode. (iii) Deflect mode, to the left. (iii') Deflect mode, to the right. (B)

Pitch-roll cross-section at different $x$ for analyzing pitch-to-roll transition barrier. Blue points are pitch local minimum and saddle point overlap. (ii) $x=-13.6 \mathrm{~mm}$. Pitch minimum and saddle point overlap. (iii) $x=0$ mm. (C) Yaw cross-sections stacked along head flexion dimension for different $x$ for analyzing between-

383 gap-to-deflect transition barrier. Blue points are between-gap local minima, the one with large body yaw is deflecting to the left. Red points are deflect local minima. Orange points are saddle points. Green curves: 
imaginary routes. Only routes at head flexion $\beta_{\mathrm{h}}=15^{\circ}$ are marked. (i) $x=-20.4 \mathrm{~mm}$. (ii) $x=-13.2 \mathrm{~mm}$. (iii) $x=-1 \mathrm{~mm}$.

\subsection{Test usefulness of head flexion}

To test hypothesis (1), we analyzed whether head flexion in the range observed reduced the pitchto-roll transition barrier compared to if the animal simply held its head in the typical orientation (average head flexion during running on flat ground). We varied head flexions within $\left[-25^{\circ}, 65^{\circ}\right]$ (covering the observed head flexion range of $\left[-24^{\circ}, 64^{\circ}\right]$ over all the trials) with an increment of $5^{\circ}$ (Fig. S2A), calculated the pitch-to-roll transition barrier as a function of $x$, and compared the transition barrier at each head flexion with that at head flexion $\beta_{\mathrm{h}}=15^{\circ}$, which represents the case without active head flexion (temporal average of head flexion was $\beta_{\mathrm{h}}=15^{\circ} \pm 4^{\circ}$ in the approach phase over all trials).

To test hypothesis (2), we analyzed whether the head flexion in the range observed increased the between-gap-to-deflect transition barrier compared to without active head flexion. We varied the head flexion like above, calculated the between-gap-to-deflect transition barrier as a function of $x$, and compared the transition barrier at each head flexion with that at head flexion $\beta_{\mathrm{h}}=15^{\circ}$.

\subsection{Test usefulness of leg sprawl reduction}

To test hypotheses (3) and (4), we analyzed whether the leg sprawl changes affected the betweengap-to-deflect transition barrier. We added two hind legs $($ length $=27 \mathrm{~mm})$ into the model symmetrically to the left and right sides of the body, with a leg height of $-5 \mathrm{~mm}$ (temporal average of leg height was $-5 \pm$ $3 \mathrm{~mm}$ in the explore + pitch phase (Sec. 3.1) over all trials), and varied leg sprawl angles within $\left[0^{\circ}, 159^{\circ}\right]$ with an increment around $10^{\circ}$ (Fig. S2B). We compared the transition barrier at each leg sprawl angle with that at leg sprawl $\phi=143^{\circ}$ (average maximal leg sprawl was $\phi=140^{\circ} \pm 18^{\circ}$ in the explore + pitch phase over all trials) and $\phi=39^{\circ}$ (average minimal leg sprawl was $\phi=40^{\circ} \pm 18^{\circ}$ in the roll phase over all trials). 


\section{Results}

\subsection{Animal uses complex motion to transition from pitch to roll mode}

After running with an alternating tripod gait and collided with the beams (approach phase, Fig. 6,

411 gray), the animal traversed the beam obstacles with complex body, head, and leg motions. In the explore +

412 pitch phase (Fig. 6, blue), the animal often moved along the beam layer ( $y$-direction) and turned left or right

413 to search around the beams, pitched up its body against the beams, flexed its head up and down, and rubbed

414 its pronotum against the beams, sometimes pushed the beam using its fore or middle legs, and swept its

415 antennas in the gaps. In the roll phase (Fig. 6, red), the animal rolled its body into the gap, flexed its head

416 repeatedly, sometimes flexed and twisted its abdomen, and struggling its legs to try to push the back surface

417 of the beam. In the land phase (Fig. 6, orange), the animal passed through the beams in the roll mode, and

418 resumed an upright body orientation. In the depart phase (Fig. 6, green), the animal ran away in an

419 alternating-tripod gait. These observations were consistent with those in the previous study (Othayoth et

420 al., 2020). The average animal trajectory as a function of forwarding position $x$ is shown in (Fig. S2).

Similar to the previous study, body oscillation was observed (Othayoth et al., 2020). The average

kinetic energy fluctuation was $0.01 \pm 0.01 \mathrm{~mJ}$. This is smaller than that in the previous study $(0.02 \pm 0.01$

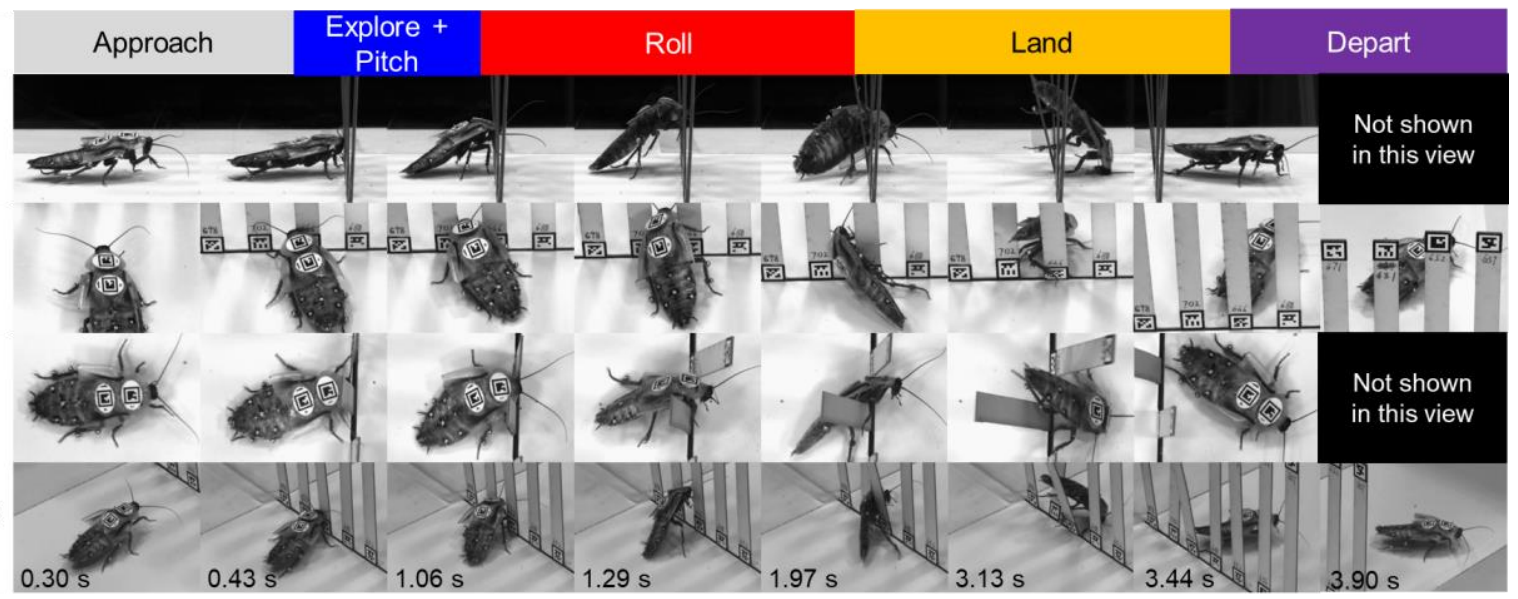




\section{Fig. 6. Five phases of traversal.}

\subsection{Body rotations}

The animal's body pitched up in the explore + pitch phase, and rolled while pitched down in the

429 roll phase. In the roll phase, the animal rolled its body significantly (maximum roll $\gamma=81^{\circ} \pm 10^{\circ}$ ) (Fig. 7A).

430 Before the animal rolled, its body pitch increased (maximum pitch $\beta=36^{\circ} \pm 9^{\circ}$ ). Then, as the animal rolled,

431 body pitch decreased (Fig. 7C). The average body roll in the roll phase was higher than in the other three

432 phases $(P<0.0001$, repeated-measures ANOVA, Fig. 7B $)$. Average body pitch in the explore + pitch phase

433 was higher than in the approach and depart phases $(P<0.0001$, repeated-measures ANOVA, Fig. 7D) and

434 was slightly higher in the roll phase than in the approach and depart phases $(P<0.05$, repeated-measures

435 ANOVA, Fig. 7D). 
A

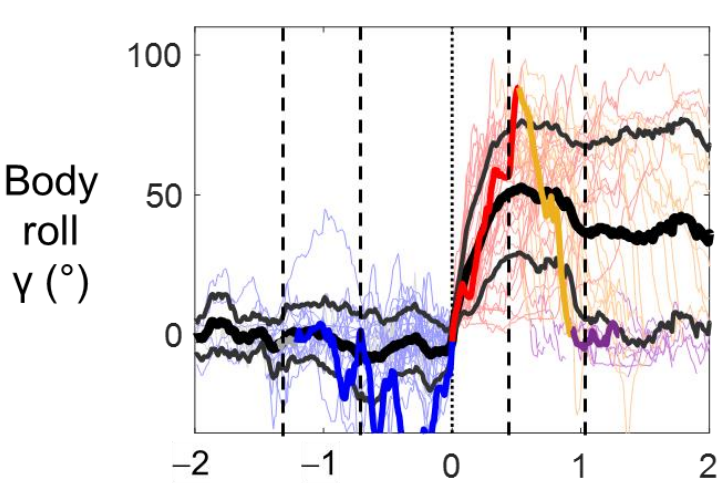

C

Body pitch $\beta\left({ }^{\circ}\right)$

$$
40
$$
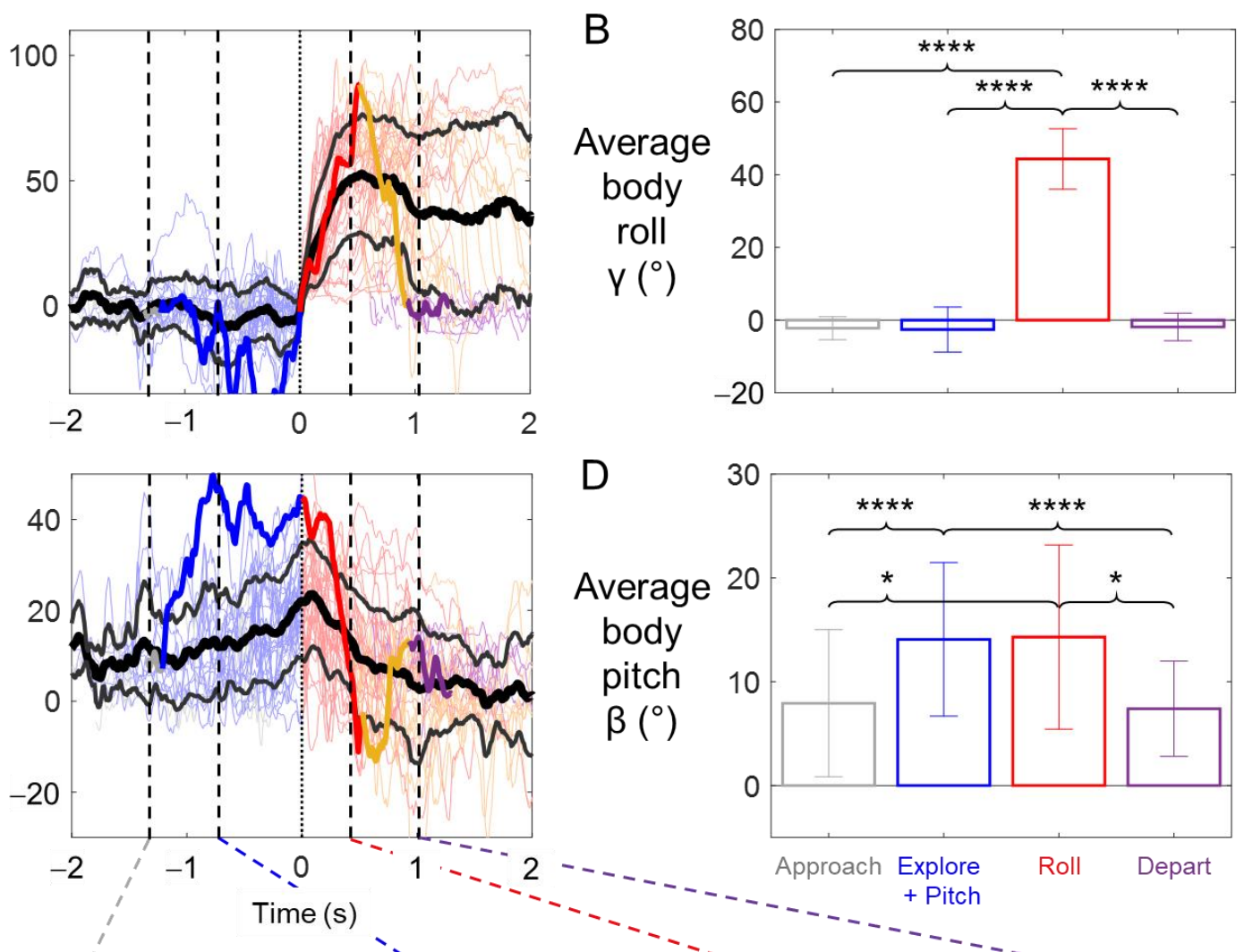

\section{E}
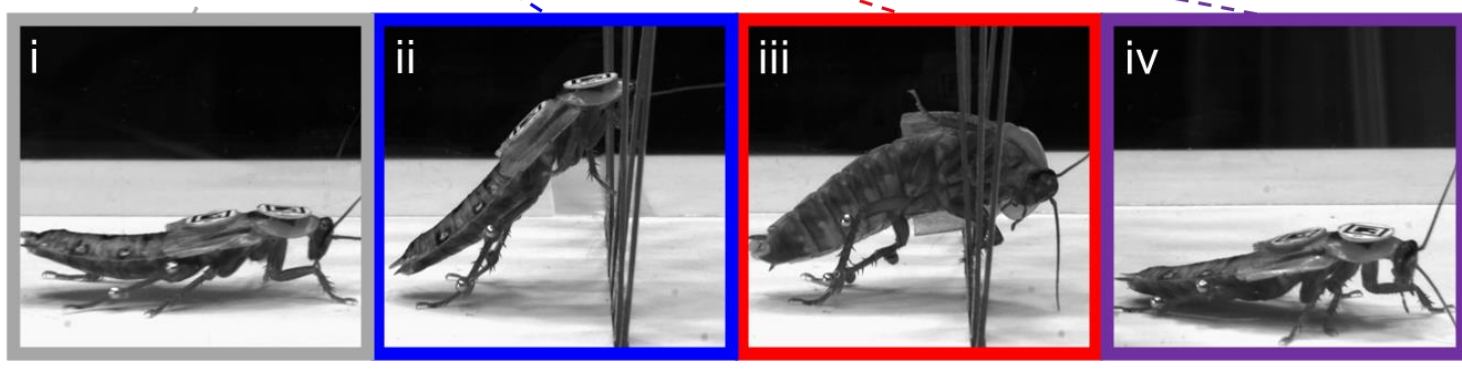

Fig. 7. Body rotations. (A) Body roll as a function of time. (B) Average body roll in different phases. (C)

Body pitch as a function of time. (D) Average body pitch in different phases. Colors are for five phases defined in Fig. 6. In (A, C), thin colored curves are individual trials; thick and thin black curves are mean \pm s.d. across all trials, and thick colored curve is an example individual trial. In (B, D), bars and error bars

441 are means \pm s.d. of the temporal averages of all trials in $(\mathrm{A}, \mathrm{C})$ for each phase. (E) Representative snapshots 442 for each phase.

\section{3.3. Head flexion}

During the explore + pitch and roll phase, the animal sometimes flexed its head up and down dynamically and sometimes flexed its head down and held it statically. (Fig. 8A, Movie 1). The standard 
A

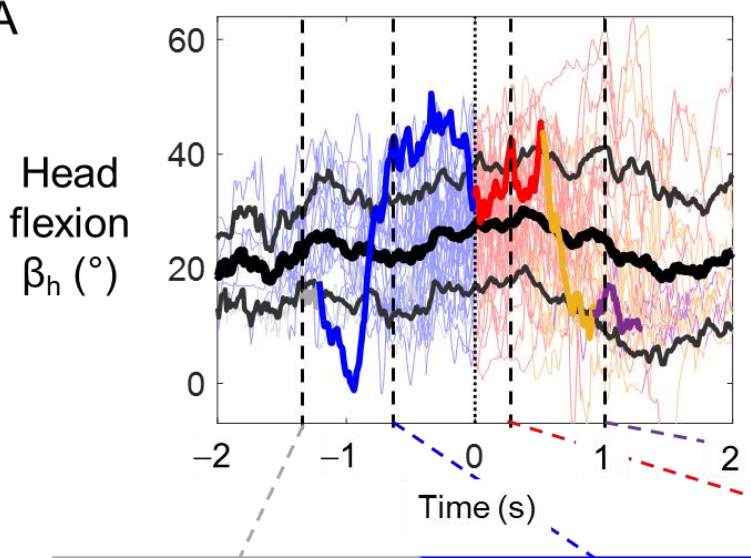

B

Average 10

s.d. of head flexion $\beta_{\mathrm{h}}\left({ }^{\circ}\right)$

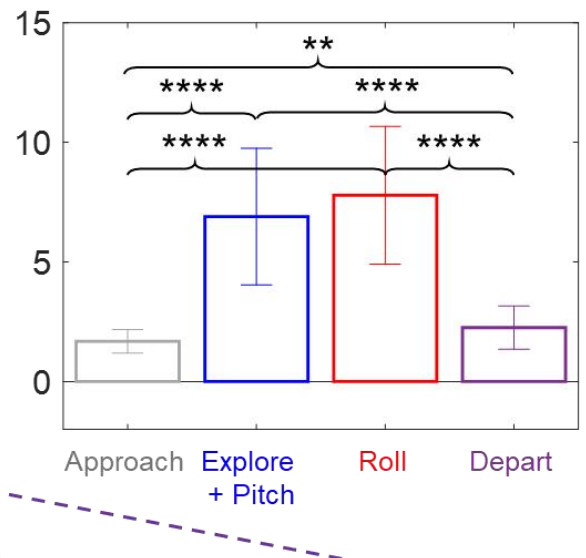

C
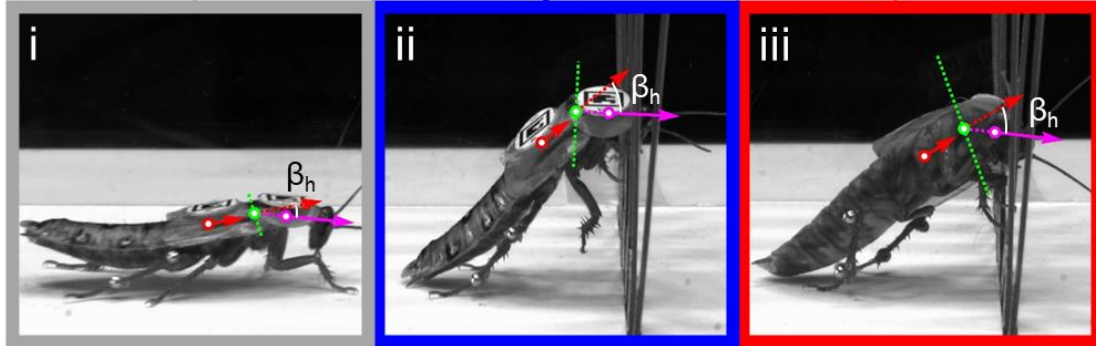

iv

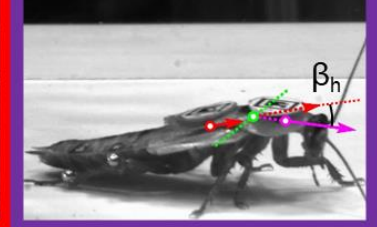

Fig. 8. Head flexion. (A) Head flexion as a function of time. Thin colored curves are individual trials; thick and thin black curves are mean \pm s.d. across all trials. (B) Average standard deviation of head flexion in different phases. Bars and error bars are means \pm s.d. of the temporal standard deviations of data in (A) for each phase. (C) Representative snapshots for each phase.

\subsection{Abdomen flexion}

The animal flexed its abdomen at a larger amplitude in the roll phase than in the other phases (Movie 2). In the approach and explore + pitch phase, the animal's abdomen only flexed down slightly (average abdomen flexion $\beta_{\mathrm{a}}=7^{\circ} \pm 4^{\circ}$ ). As the body began to roll, abdomen flexion increased (maximum abdomen flexion $\beta_{\mathrm{a}}=37^{\circ} \pm 12^{\circ}$ ) (Fig. 9A). Average abdomen flexion was higher in the roll phase than in the three other phases $(P<0.0001$, repeated-measures ANOVA, Fig. 9B) and was slightly higher in the 
Fig. 9. Abdomen flexion statistics. (A) Abdomen flexion as a function of time. Thin colored curves are each phase. (C) Representative snapshots for each phase.

\subsection{Leg adjustments}

The animal adjusted its hind legs in the roll phase in two ways (Movie 3). First, it tucked its hind (average leg sprawl $=115^{\circ} \pm 16^{\circ}$ ). As rolling began, the animal tucked its hind legs inward (average leg sprawl $\left.=84^{\circ} \pm 15^{\circ}\right)($ Fig. 10A). Average leg sprawl was larger in the explore + pitch phase than in the other other three phases $(P<0.0001$, repeated-measures ANOVA, Fig. 10B). Average leg sprawl was slightly different in the depart phase from the approach or the explore + pitch phase $(P<0.05$, repeated-measures ANOVA, Fig. 10B).

477 roll phase, one hind leg extended (maximum leg heights $=19 \pm 3 \mathrm{~mm}$ ), appearing to push against the ground, while the other retracted (minimum leg heights $=-4 \pm 2 \mathrm{~mm}$ ), which increased the leg height

481 higher in the roll phase than in the other three phases $(P<0.0001$, repeated-measures ANOVA) (Fig. 10D).

482 Both these observations demonstrated that the animal used its legs differentially in the roll phase compared 483 with the other three phases. 
bioRxiv preprint doi: https://doi.org/10.1101/2021.10.02.462900; this version posted October 4, 2021. The copyright holder for this preprint (which was not certified by peer review) is the author/funder, who has granted bioRxiv a license to display the preprint in perpetuity. It is made available under aCC-BY-NC-ND 4.0 International license.

A

Leg sprawl

$\phi\left(^{\circ}\right)$

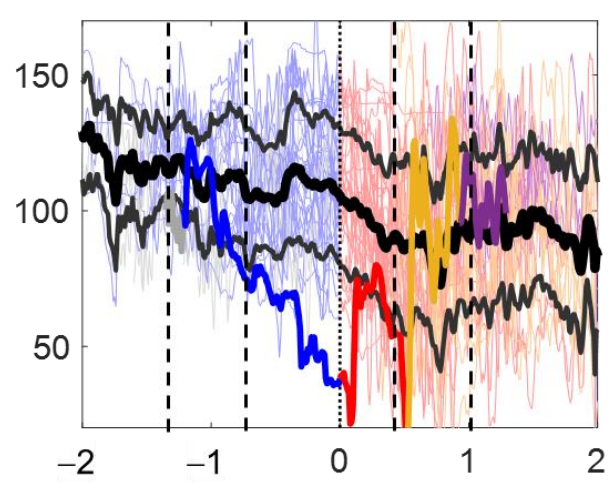

C

Leg height $h(\mathrm{~mm})$

$E$
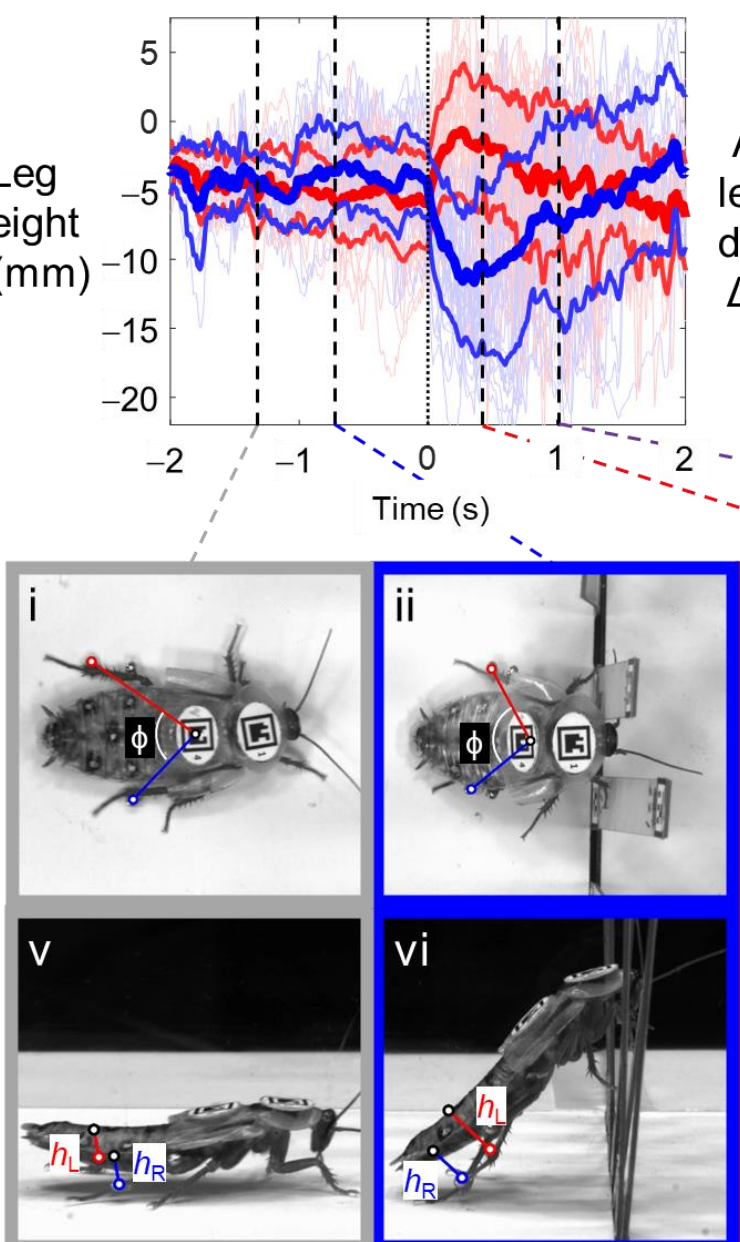

$\mathrm{D}$

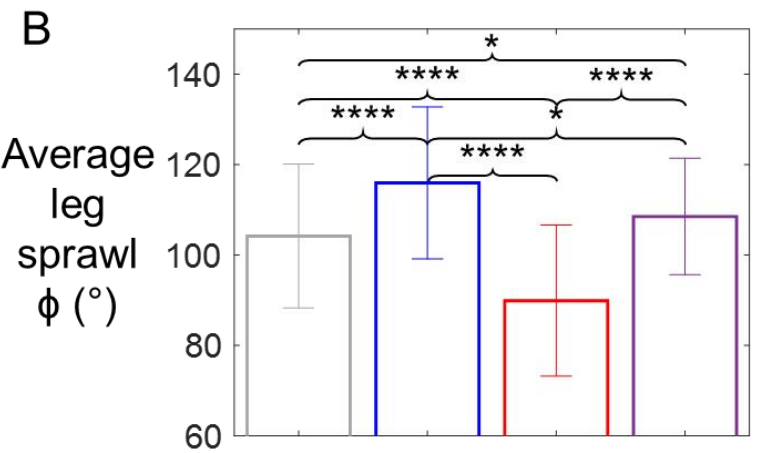

D 20

Average leg height 10 difference 5 $\Delta h(\mathrm{~mm})$

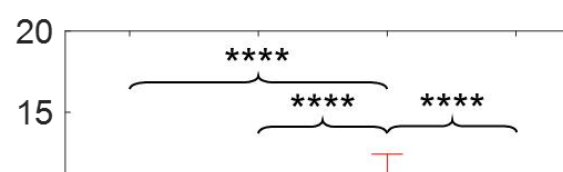

5
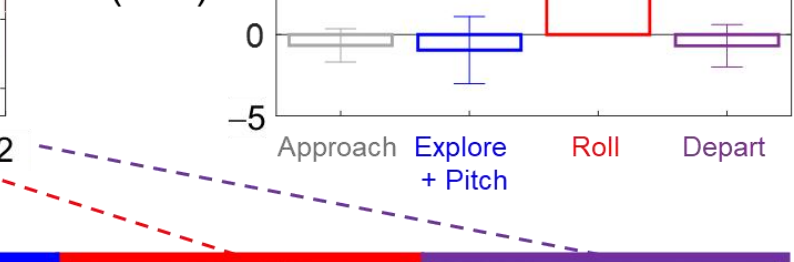

+ Pitch
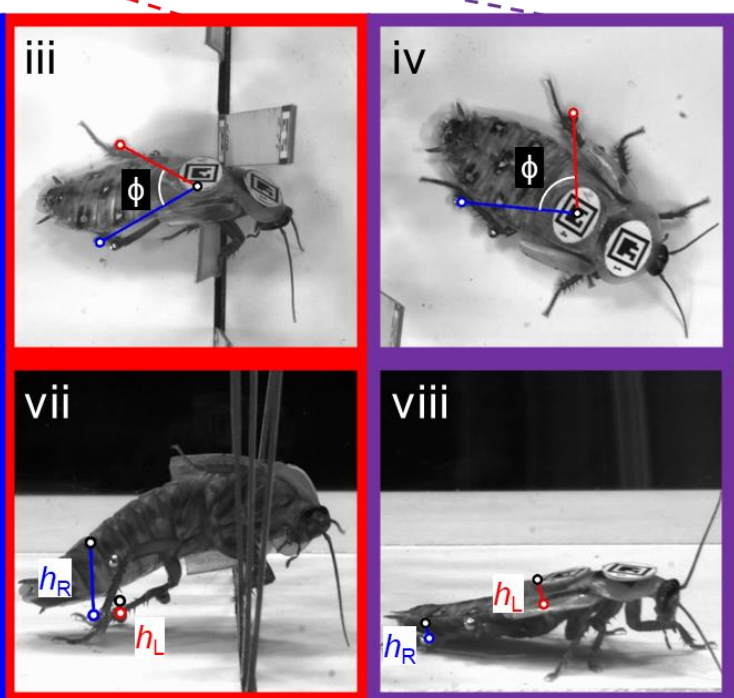

Fig. 10. Leg adjustments statistics. (A) Leg sprawl as a function of time. Thin colored curves are individual trials; thick and thin black curves are mean \pm s.d. across all trials. (B) Leg sprawl in different

487 phases. (C) Leg height of hind legs as a function of time. Blue: left leg. Red: right leg. Light-colored curves are individual trials; thick and thin black and solid colored curves are mean \pm s.d. across all trials. (D) Leg 
height difference in different phases. In (B, D), bars and error bars are means \pm s.d. from temporal averaging of data in $(\mathrm{A}, \mathrm{C})$ for each phase. $(\mathbf{E})$ Representative snapshots for each phase.

\subsection{Refined potential energy landscape consistent with coarse landscape in previous study}

The topology and evolution of the refined potential energy landscape as viewed in the pitch-roll cross-section was consistent with that in the previous study (Othayoth et al., 2020). Initially, when the animal was far from the beam, the energy landscape had a local minimum at zero pitch and roll, a basin was formed near the local minimum; as the body moved close to the beam, the basin moved along pitch direction, becoming the pitch basin, while the roll basin formed at about zero pitch and about $90^{\circ}$ roll. Because we allowed the beam to deflect backward, the pitch basin finally went back to near zero pitch, and traversed and moved far from the beam. the initial landscape showed that the beam deflection calculation was more reasonable compared to the previous study.

\subsection{Head flexion does not facilitate pitch-to-roll transition}

To test hypothesis (1), we analyzed whether adjusting head flexion can reduce the pitch-to-roll 
514 to-deflect transition barrier. The transition barrier from the between-gap to the deflect mode with different

515 head flexion is shown in Fig. 11B, C. At the average $x(-13.6 \mathrm{~mm})$ where the animal transitioned from the

516 pitch to the roll mode, the maximal increase in transition barrier with head flexion within $\left[-25^{\circ}, 65^{\circ}\right]$ was

$517 \quad 0.012 \mathrm{~mJ}$ to deflect to the left and $0.008 \mathrm{~mJ}$ to deflect to the right. The transition barrier only increased by $51810 \%$, which required the head to hyperextend (head flexion $\beta_{\mathrm{h}}=-25^{\circ}$ ), which was rarely observed in the

519 experiment. This suggested that the head adjustment did not increase the transition barrier substantially to

520 prevent the animal from yawing and deflecting the beams. This rejected our hypothesis that the head 521 adjustment facilitates the body staying within the gap.

\section{$522 \quad$ 3.8. Leg sprawl adjustments facilitate body rolling}

To test hypotheses (3) and (4), we analyzed whether adjusting leg sprawl increases or reduces the

524 pitch-to-roll transition barrier. The transition barrier from pitch to roll mode with different leg sprawl is

525 shown in Fig. 11D. At $x=-20 \mathrm{~mm}$ where the animals pitched against the beams (temporal average of $x$

526 was $-20 \pm 3 \mathrm{~mm}$ in the pitch + explore phase over all trials), the transition barrier at $143^{\circ}$ leg sprawl was

527 larger than that at $39^{\circ}$ leg sprawl by $0.32 \mathrm{~mJ}$, which was 32 times larger than the average kinetic fluctuation

$528(0.01 \mathrm{~mJ})$. Because a larger potential energy barrier suggests higher stability, this indicated that a large leg

529 sprawl helped the animal stay pitched up against the beams stably. At $x=-13.6 \mathrm{~mm}$ where the animals

530 transitioned from the pitch to the roll mode, the transition barrier at $39^{\circ}$ leg sprawl was less than that at

$531143^{\circ}$ leg sprawl by $0.03 \mathrm{~mJ}$, which is 3 times larger than the average kinetic fluctuation level. These

532 indicated that a small leg sprawl angle helped the animal reduce the pitch-to-roll transition barrier when

533 rolling. Together, these findings supported our hypothesis that leg sprawl adjustment facilitated pitch-to-

534 roll transition. 
A

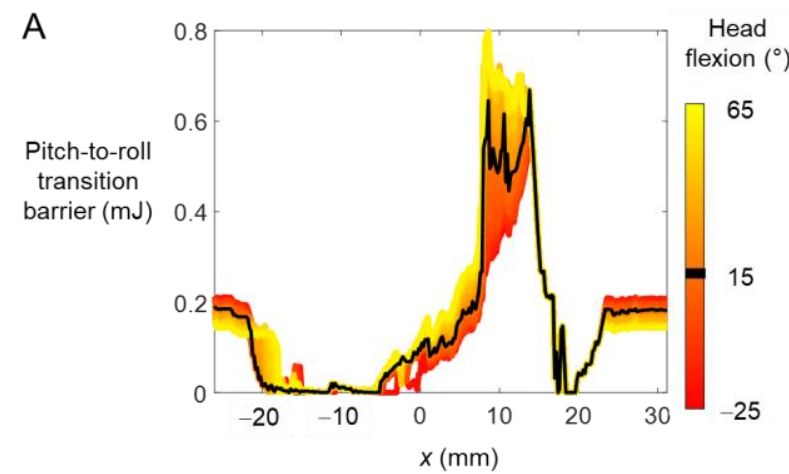

C

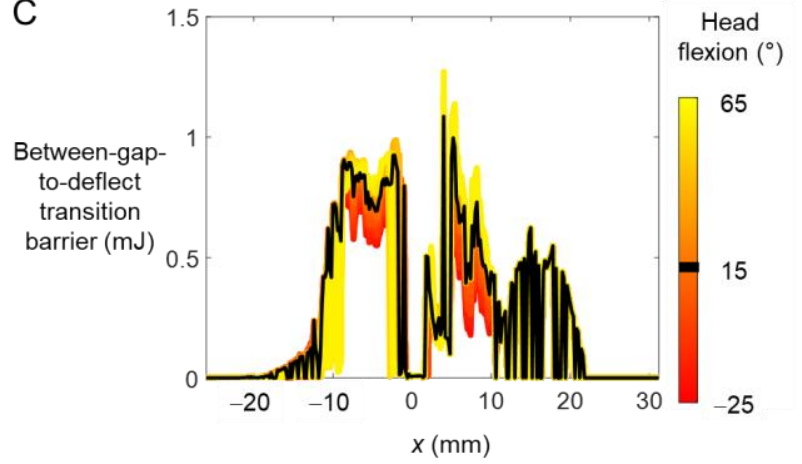

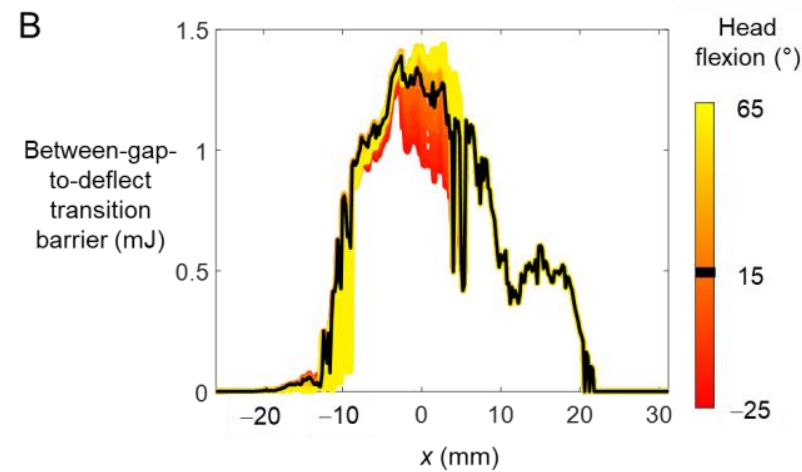

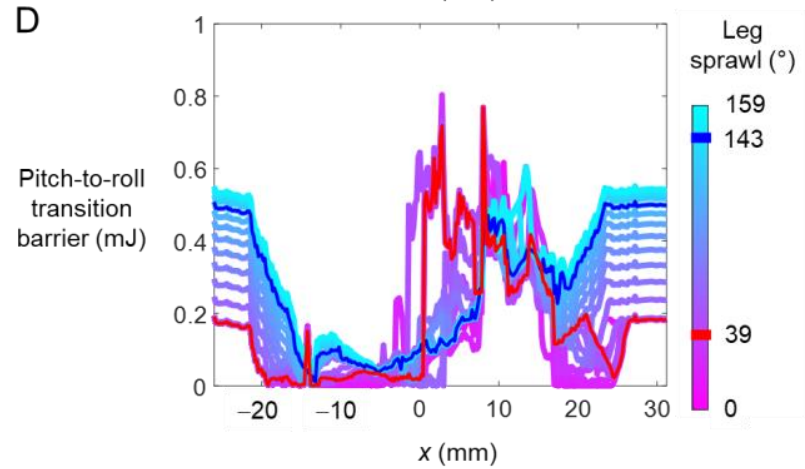

Fig. 11. Effect of head flexion on transition barriers. (A) Pitch-to-roll transition barrier as a function of $x$ for different head flexion. (B, C) Roll-to-deflect transition barrier as a function of $x$ under for different head flexion. (B) deflect to the left. (C) deflect to the right. In (A, B, C), black curves show transition barrier at average animal head flexion $\beta_{\mathrm{h}}=15^{\circ}$. (D) Pitch-to-roll transition barrier as a function of $x$ for different leg sprawl angles. Red and blue curves show transition barrier at leg sprawl $\phi=39^{\circ}$ and $143^{\circ}$, respectively.

\section{Discussion}

As a first step to understand how animals actively control physical interaction with complex 3-D terrain to transition between locomotor modes, we quantified active adjustments by the discoid cockroach

547 Because it is strenuous to traverse the stiff beams by pushing across (the pitch mode), the animal likely made these active adjustments to facilitate transitioning to the less strenuous roll mode. Below we discuss 
the likely function of each adjustment and suggest future directions.

\subsection{Role of head flexion}

To find the function of active head flexion in both the explore + pitch and roll phases, we first hypothesized that by changing the overall body shape, it (1) lowered the pitch-to-roll transition barrier and (2) increased the between-gap-to-deflect transition barrier. However, we found that the head flexion did not change the transition barrier substantially in both cases. Therefore, we rejected these two hypotheses (Sec. 3.7). We speculate that this is because the cockroach's head is small and relatively more spherical (thickness/length $=0.66)$ compared to the thorax $(0.30)$ and abdomen $(0.21)$, so its orientation does not change overall body shape substantially, resulting in too small of a change in the potential energy landscape.

We speculate that the animal flexed its head to sense obstacle properties in the explore + pitch phase. Groups of campaniform sensilla and sensory hairs embedded in the cockroach's pronotum can sense the magnitude, direction, and position of the terrain reaction force (Delcomyn et al., 1996). The chordotonal organ in the animal's neck can detect the forces pushing against the head (Field and Matheson, 1998; Tuthill and Wilson, 2016). These could help the animal estimate the obstacle's physical properties (stiffness, surface friction coefficient, etc.) (Xuan et al., 2021) and guide its active adjustments to better traverse. We speculate that the occasional dynamically changing head flexion is a form of active sensing (Cellini et al., 2021; Towal, 2006). We observed that the animal seemed to flex the head upward in order to find the top end of the stiff beam obstacles (to initiate climbing) and flexed the head downward in order to find a gap to move into.

In addition, we speculate that the animal flexed its head in the later part of the roll phase (after the center of mass has passed the beams) to help its fore legs reach the ground to help propel forward, while its middle and hind legs were still interacting with the beams and were likely less effective at generating propulsion within the narrow gap. This is similar to cockroaches flexing the head to help fore legs reach the top surface when climbing a large step (Ritzmann et al., 2005).

\subsection{Role of abdomen flexion}


We speculate that the animal flexed its abdomen frequently in the roll phase to generate kinetic energy fluctuation to break resistive frictional and interlocking contact as it pushed through the beams when rolled. Because the beam gap was narrow and barely larger than the animal body thickness (average body thickness/gap width $=73 \%$, not including legs), the animal had to retract its legs closer to the body (Fig.

578 12B, iv) to fit them within the gap. This made it difficult to generate thrust force from the legs. Meanwhile,

579 the spines and other asperities on the thorax, abdomen, and legs added resistance, similar to a cockroach

580 crawling in a confined space (Jayaram and Full, 2016). This kinetic energy fluctuation from abdomen

581 flexion helps the animal become unstuck and thus facilitates traversal.

\subsection{Role of leg adjustments}

We speculated that the animal spread its hind legs outward to stabilize the pitched-up body against the beams in the pitch + explore phase and it tucked the hind legs inward to facilitate rolling in the roll phase. Geometrically, the stable support polygon of the animal was formed among contacts between its hind feet and the ground and contacts between its head or thorax and the beams. In the explore + pitch phase, the animal's hind legs sprawled out widely, with a large distance between the hind feet touching the ground, which increased the animal's roll stability (Fig.12A). In the roll phase, as the animal tucked its legs

591 from the pitch to the roll basin measures the difficulty to transition. In the explore + pitch phase, a large leg 592 sprawl increased the transition barrier, which helped the animal stay pitched up against the beams. During 593 pitch-to-roll transition, a small leg sprawl reduced the transition barrier and made rolling easier.

A
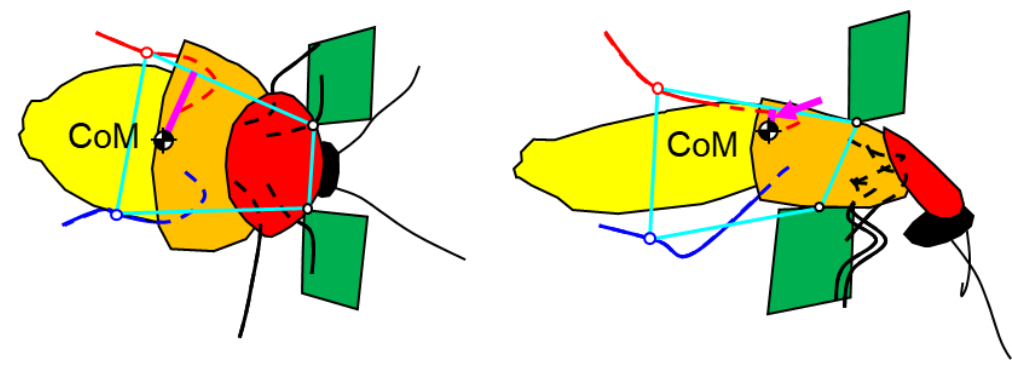
Fig. 12. Representative support polygon evolution during pitch-to-roll transition. (A) Explore + pitch from center of mass $(\mathrm{CoM})$ to nearest lateral edge of the support polygon, which measures roll stability. In (B), the distance is small and a magenta arrow is drawn.

Aside from leg sprawl, the differential leg uses also played an important role. We speculated that the animal extended one hind leg and retracted the other in the roll phase to generate a roll torque and it retracted both hind legs in the later part of the roll phase to reduce the resistance on the legs from the beams.

\subsection{Role of body flexibility} resistance and facilitated traversal.

\subsection{Likely involvement of sensory feedback control}

Studies on insects negotiating large obstacles have revealed that the changes in kinematics are

611 modulated by sensory inputs. For example, when climbing large stairs, stick insects switch from using long

612 to short steps when they have sensed a lack of substrate engagement (Theunissen and Dürr, 2013); when

613 climbing a large step, cockroaches flex the head to help fore legs reach the top step surface when its head

614 has sensed that it has risen above the step (Ritzmann et al., 2005). During traversal of cluttered beams in

615 our study, the cockroach's active adjustment to make the pitch-to-roll transition is almost certainly also

616 driven by sensory input of its environment-interaction. During traversal, it took the animal an average time

617 of $1.3 \pm 0.7 \mathrm{~s}$ to explore and pitch up against the beams before rolling occurred. This is well above the $\sim 100$

618 ms that cockroaches need to complete a feedback control loop (6-40 ms for the sensory delay (Ritzmann et 

transitioning to the less strenuous roll mode. We have recently explored the feasibility of this in a simple

622 simulated robotic physical model (Xuan et al., 2021).

\subsection{Future work}

Future work should verify the speculations of the mechanisms how each kind of adjustment facilitates the mode transition. (1) To understand how the flexing head facilitates sensing and how to take advantage of this, we can build a robot that is capable of the functions of head and abdomen flexing and whether the abdomen flexion helps the animal become unstuck, we can build a simple robot with a flexing abdomen or tail to test if the flexing of the massive lateral part helps in beams traversal. (3) To test whether

630 active leg adjustments indeed generates a roll torque, we can add separated force plates (Goldman et al., 631 2006) to the ground in front of the beams to sense the ground reaction force on each foot.

To further understand the neural mechanisms involved in such cluttered large obstacle traversal, we should measure the animal's sensory neural signals (Mongeau et al., 2015; Ritzmann et al., 2012) and muscle activity (electromyogram) (Sponberg and Full, 2008; Watson et al., 2002) and alter motor activation 
644 disabling the campaniform sensilla (Pearson and Iles, 1973) and observe changes in locomotor behavior

645 and performance. Based on animal observations, computational modeling of neural control (such as in

646 (Schilling and Cruse, 2020)) may be fruitful for understanding feedback principles governing body and

647 appendage adjustments to traverse cluttered large obstacles. In addition, it may be interesting to study

648 whether animals perform active sensing (Krakauer et al., 2017; Mongeau et al., 2013; Moss et al., 2006;

649 Nelson and MacIver, 2006; Okada et al., 2002; Schütz and Dürr, 2011; Stamper et al., 2012) in the less650 considered modality of contact force sensing.

Our case study illustrated how to use fine-grained potential energy landscape modeling to

654 (Othayoth et al., 2020)). Fundamental insights from our study may also guide the development of multi655 legged robots that can traverse cluttered terrain like forest floor for environmental monitoring (Matsuno et al., 1998), earthquake rubble for search and rescue (Freitas et al., 2011), and Martian rocks for planetary exploration (Matthies et al., 2007).

\section{Acknowledgments}

We thank Yuanfeng Han and Qiyuan Fu for help with imaging setup, Yuanfeng Han and Qihan

Xuan for discussion, and Xiao Yu for help with animal care and manually correcting the marker tracking.

\section{Competing interests}




\section{Author contributions}

Conceptualization: C.L.; Methodology: Y.W., R.O., C.L.; Software: Y.W, R.O.; Validation: Y.W.,

674 Writing - original draft: Y.W.; Writing - review \& editing: Y.W., R.O., C.L.; Visualization: Y.W.;

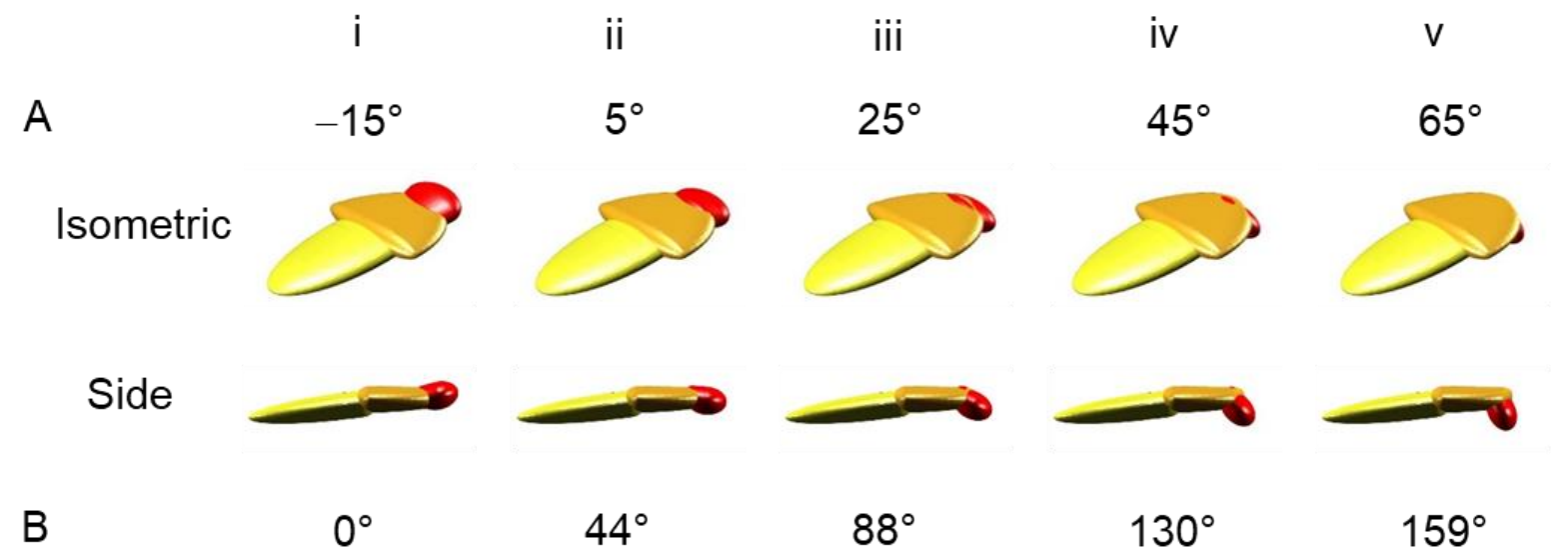

B

$0^{\circ}$

$44^{\circ}$

$130^{\circ}$

$159^{\circ}$

Fig. S1. Example variation of head flexion and hind leg sprawl to test the use of head and leg 


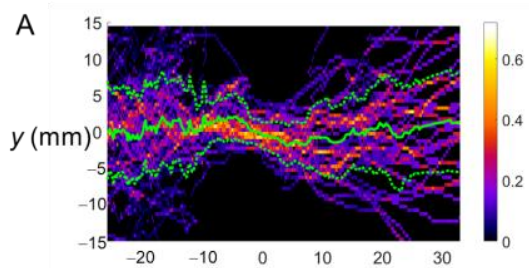

D

Ditch
$\beta\left({ }^{\circ}\right)$

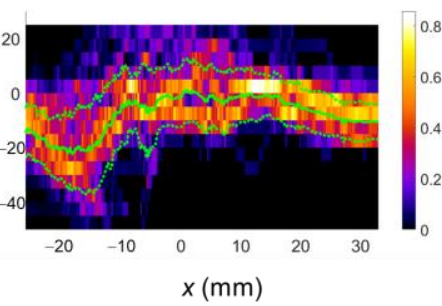

B

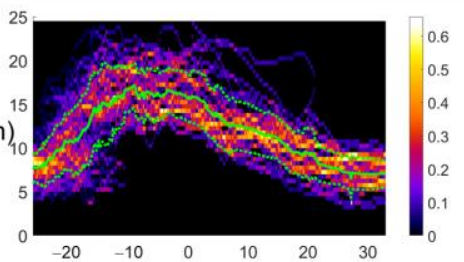

E $\quad 100$

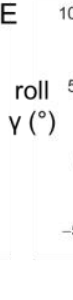

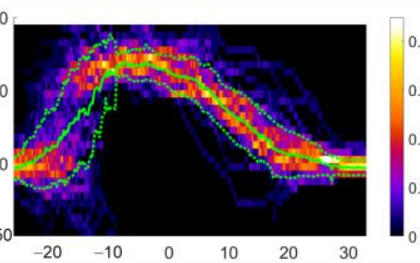

$x(\mathrm{~mm})$
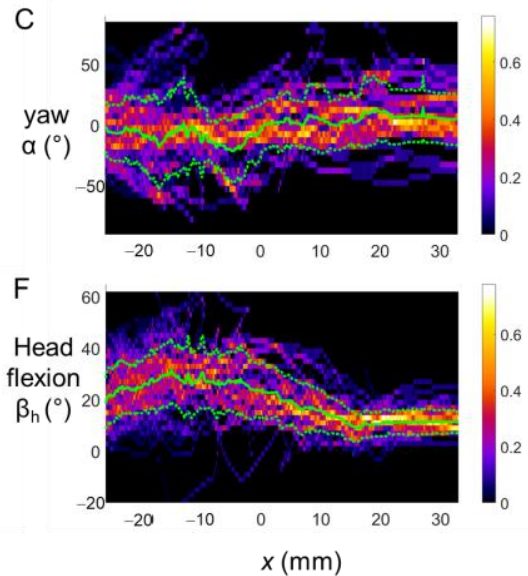

Fig. S2. Average trajectory as a function of forwarding position $x$. (A) Lateral position $y$. (B) Vertical

mean \pm s.d. from averaging data of all trials.

Table S1. Frequently used averaged variables and range

\begin{tabular}{|c|c|c|c|c|}
\hline Variable & Time range & Measured value & Used value & First used in Sec. \\
\hline Forward position $x$ & Pitch-to-roll transition & $-13.6 \pm 4.4 \mathrm{~mm}$ & $-13.6 \mathrm{~mm}$ & 2.9 \\
\hline Maximum leg length & $\begin{array}{c}\text { Explore + pitch and } \\
\text { roll phase }\end{array}$ & $27 \pm 2 \mathrm{~mm}$ & $27 \mathrm{~mm}$ & 2.9 \\
\hline TA abdomen flexion $\beta_{\mathrm{a}}$ & Approach phase & $7^{\circ} \pm 4^{\circ}$ & $7^{\circ}$ & 2.10 \\
\hline Head flexion range $\beta_{\mathrm{h}}$ & Whole trial & {$\left[-24^{\circ}, 64^{\circ}\right]$} & -12 \\
\hline TA head flexion $\beta_{\mathrm{h}}$ & Approach phase & $15^{\circ} \pm 4^{\circ}$ & $15^{\circ}$ & 2.12 \\
\hline TA leg height & Explore + pitch phase & $-5 \pm 3 \mathrm{~mm}$ & $-5 \mathrm{~mm}$ & 2.13 \\
\hline Maximum leg sprawl $\phi$ & Explore + pitch phase & $140^{\circ} \pm 18^{\circ}$ & $143^{\circ}$ & 2.13 \\
\hline Minimum leg sprawl $\phi$ & Explore + pitch phase & $40^{\circ} \pm 18^{\circ}$ & $39^{\circ}$ & 3.1 \\
\hline Kinetic energy & Explore + pitch, roll & $0.01 \pm 0.01 \mathrm{~mJ}$ & $0.01 \mathrm{~mJ}$ & \\
\hline fluctuation & and land phase & & $-20 \mathrm{~mm}$ & 2.8 \\
\hline TA forward position $x$ & Explore + pitch phase & $-20 \pm 3 \mathrm{~mm}$ & \\
\hline
\end{tabular}


TA: temporal averaged

\section{Supplementary Movies}

689 Movie 1. Head flexion. Top: zoomed top (left) and side (right) views. White points with red, magenta, and 690 green edges are origin of body frame, origin of head frame, and middle point of thorax-head joint, 691 respectively. Solid and dotted arrows show $+x$ and $+x$ ' direction of body (red) and head (magenta) frames, 692 respectively. Head flexion is the angle between these two directions. Bottom left: isometric view. Bottom 693 right: head flexion as a function of time.

694 Movie 2. Abdomen flexion. Top: zoomed top (left) and side (right) views. White points with red, magenta, and green edges are origin of body frame, origin of abdomen frame, and middle point of the thorax-abdomen

697 frames, respectively. Abdomen flexion is the angle between these two directions. Bottom left: isometric 698 view. (D) abdomen flexion as a function of time.

699 Movie 3. Leg adjustments. Top left: zoomed top view. White points with red, blue, and black edges are the 700 left and right tibia-tarsal joints and origin of body frame, respectively. Leg sprawl is the angle between the 701 solid red and blue lines. Top right: zoomed side view. White points with red, blue, and black edges are 702 tibia-tarsal joints and their projections to body coronal plane, respectively. Leg height of left and right hind 703 legs is the length of the red and blue lines, respectively. Bottom left: isometric view. Bottom right: Leg 704 sprawl (top) and leg height (bottom) as a function of time. Red and blue are for left and right hind legs, 705 respectively.

\section{References}

708 Alexander, R. M. (2006). Principles of animal locomotion. Princeton, NJ: Princeton University Press.

709 Allen, T. J., Quinn, R. D., Bachmann, R. J. and Ritzmann, R. E. (2003). Abstracted biological

710 principles applied with reduced actuation improve mobility of legged vehicles. In Proceedings 2003 
IEEE/RSJ International Conference on Intelligent Robots and Systems (IROS 2003) (Cat. No.03CH37453), pp. 1370-1375. IEEE.

Blaesing, B. and Cruse, H. (2004). Stick insect locomotion in a complex environment: climbing over large gaps. J. Exp. Biol. 207, 1273-1286.

Blickhan, R. and Full, R. J. (1993). Similarity in multilegged locomotion: Bouncing like a monopode. $J$. Comp. Physiol. A 173, 509-517.

Bramble, D. M. and Lieberman, D. E. (2004). Endurance running and the evolution of Homo. Nature 432, 345-352.

Cellini, B., Salem, W. and Mongeau, J.-M. (2021). Mechanisms of punctuated vision in fly flight. Curr. Biol. 31, 4009-4024.e3.

Cormen, T. H., Leiserson, C. E., Rivest, R. L. and Stein Clifford (2009). Introduction to algorithms. third edit. The MIT Press.

Crall, J. D., Gravish, N., Mountcastle, A. M. and Combes, S. A. (2015). BEEtag: A Low-Cost, ImageBased Tracking System for the Study of Animal Behavior and Locomotion. PLoS One 10, e0136487.

Delcomyn, F., Nelson, M. E. and Cocatre-Zilgien, J. H. (1996). Sense Organs of Insect Legs and the Selection of Sensors for Agile Walking Robots. Int. J. Rob. Res. 15, 113-127.

Dickinson, M. H. (2000). How Animals Move: An Integrative View. Science (80-. ). 288, 100-106.

Field, L. H. and Matheson, T. (1998). Chordotonal Organs of Insects. In Advances in Insect Physiology, pp. 1-228.

Freitas, G., Lizarralde, F., Hsu, L., Paranhos, V., Reis, N. R. S. dos and Bergerman, M. (2011). Design, Modeling, and Control of a Wheel-Legged Locomotion System for the Environmental Hybrid Robot. In Biomechanics / 752: Robotics, pp. 302-310. Calgary,AB,Canada: ACTAPRESS.

Goldman, D. I., Chen, T. S., Dudek, D. M. and Full, R. J. (2006). Dynamics of rapid vertical climbing in cockroaches reveals a template. J. Exp. Biol. 209, 2990-3000.

Harley, C. M., English, B. A. and Ritzmann, R. E. (2009). Characterization of obstacle negotiation 
behaviors in the cockroach, Blaberus discoidalis. J. Exp. Biol. 212, 1463-1476.

Hedrick, T. L. (2008). Software techniques for two- and three-dimensional kinematic measurements of biological and biomimetic systems. Bioinspir. Biomim. 3, 034001.

Ijspeert, A. J. (2008). Central pattern generators for locomotion control in animals and robots: A review. Neural Networks 21, 642-653.

Jayaram, K. and Full, R. J. (2016). Cockroaches traverse crevices, crawl rapidly in confined spaces, and inspire a soft, legged robot. Proc. Natl. Acad. Sci. 113, E950-E957.

Krakauer, J. W., Ghazanfar, A. A., Gomez-Marin, A., MacIver, M. A. and Poeppel, D. (2017). Neuroscience Needs Behavior: Correcting a Reductionist Bias. Neuron 93, 480-490.

Kram, R., Wong, B. and Full, R. J. (1997). Three-dimensional kinematics and limb kinetic energy of running cockroaches. J. Exp. Biol. 200, 1919-1929.

Kuo, A. D. (2007). The six determinants of gait and the inverted pendulum analogy: A dynamic walking perspective. Hum. Mov. Sci. 26, 617-656.

Li, C., Pullin, A. O., Haldane, D. W., Lam, H. K., Fearing, R. S. and Full, R. J. (2015). Terradynamically streamlined shapes in animals and robots enhance traversability through densely cluttered terrain. Bioinspiration and Biomimetics 10, 46003.

Lock, R. J., Burgess, S. C. and Vaidyanathan, R. (2013). Multi-modal locomotion: from animal to application. Bioinspir. Biomim. 9, 011001.

Low, K. H., Hu, T., Mohammed, S., Tangorra, J. and Kovac, M. (2015). Perspectives on biologically inspired hybrid and multi-modal locomotion. Bioinspir. Biomim. 10, 020301.

Mathis, A., Mamidanna, P., Cury, K. M., Abe, T., Murthy, V. N., Mathis, M. W. and Bethge, M. (2018). DeepLabCut: markerless pose estimation of user-defined body parts with deep learning. Nat. Neurosci. 21, 1281-1289.

Matsuno, F., Sato, N., Kon, K., Igarashi, H., Kimura, T. and Murphy, R. (1998). Utilization of Robot Systems in Disaster Sites of the Great Eastern Japan Earthquake. (ed. Zelinsky, A.) London: Springer London. 
Matthies, L., Maimone, M., Johnson, A., Cheng, Y., Willson, R., Villalpando, C., Goldberg, S., Huertas, A., Stein, A. and Angelova, A. (2007). Computer Vision on Mars. Int. J. Comput. Vis. 75, $67-92$.

Mongeau, J. M., Demir, A., Lee, J., Cowan, N. J. and Full, R. J. (2013). Locomotion-and mechanicsmediated tactile sensing: Antenna reconfiguration simplifies control during high-speed navigation in cockroaches. J. Exp. Biol. 216, 4530-4541.

Mongeau, J.-M., Sponberg, S. N., Miller, J. P. and Full, R. J. (2015). Sensory processing within antenna enables rapid implementation of feedback control for high-speed running maneuvers. $J$. Exp. Biol. 218, 2344-2354.

Moss, C. F., Bohn, K., Gilkenson, H. and Surlykke, A. (2006). Active Listening for Spatial Orientation in a Complex Auditory Scene. PLoS Biol. 4, e79.

Nelson, M. E. and MacIver, M. A. (2006). Sensory acquisition in active sensing systems. J. Comp. Physiol. A 192, 573-586.

Okada, J., Kanamaru, Y. and Toh, Y. (2002). Mechanosensory Control of Antennal Movement by the Scapal Hair Plate in the American Cockroach. Zoolog. Sci. 19, 1201-1210.

Othayoth, R., Thoms, G. and Li, C. (2020). An energy landscape approach to locomotor transitions in complex 3D terrain. Proc. Natl. Acad. Sci. 117, 14987-14995.

Othayoth, R., Xuan, Q., Wang, Y. and Li, C. (2021). Locomotor transitions in the potential energy landscape-dominated regime. Proc. R. Soc. B Biol. Sci. 288, rspb.2020.2734.

Pearson, K. G. and Iles, J. F. (1973). Nervous Mechanisms Underlying Intersegmental Co-Ordination of Leg Movements During Walking in the Cockroach. J. Exp. Biol. 58, 725-744.

Ritzmann, R. E., Pollack, A. J., Archinal, J., Ridgel, A. L. and Quinn, R. D. (2005). Descending control of body attitude in the cockroach Blaberus discoidalis and its role in incline climbing. $J$. Comp. Physiol. A 191, 253-264.

Ritzmann, R. E., Harley, C. M., Daltorio, K. A., Tietz, B. R., Pollack, A. J., Bender, J. A., Guo, P., Horomanski, A. L., Kathman, N. D., Nieuwoudt, C., et al. (2012). Deciding Which Way to Go: 
How Do Insects Alter Movements to Negotiate Barriers? Front. Neurosci. 6, 1-10.

Schilling, M. and Cruse, H. (2020). Decentralized control of insect walking: A simple neural network explains a wide range of behavioral and neurophysiological results. PLOS Comput. Biol. 16, e1007804.

Schütz, C. and Dürr, V. (2011). Active tactile exploration for adaptive locomotion in the stick insect. Philos. Trans. R. Soc. B Biol. Sci. 366, 2996-3005.

Shepard, E. L. C., Wilson, R. P., Rees, W. G., Grundy, E., Lambertucci, S. A. and Vosper, S. B. (2013). Energy Landscapes Shape Animal Movement Ecology. Am. Nat. 182, 298-312.

Spirito, C. P. and Mushrush, D. L. (1979). Interlimb Coordination During Slow Walking in the Cockroach: I. Effects of Substrate Alterations. J. Exp. Biol. 78, 233-243.

Sponberg, S. and Full, R. J. (2008). Neuromechanical response of musculo-skeletal structures in cockroaches during rapid running on rough terrain. J. Exp. Biol. 211, 433-446.

Sponberg, S., Spence, A. J., Mullens, C. H. and Full, R. J. (2011a). A single muscle's multifunctional control potential of body dynamics for postural control and running. Philos. Trans. R. Soc. B Biol. Sci. 366, 1592-1605.

Sponberg, S., Libby, T., Mullens, C. H. and Full, R. J. (2011b). Shifts in a single muscle's control potential of body dynamics are determined by mechanical feedback. Philos. Trans. R. Soc. B Biol. Sci. 366, 1606-1620.

Stamper, S. A., Roth, E., Cowan, N. J. and Fortune, E. S. (2012). Active sensing via movement shapes spatiotemporal patterns of sensory feedback. J. Exp. Biol. 215, 1567-1574.

Theunissen, L. M. and Dürr, V. (2013). Insects Use Two Distinct Classes of Steps during Unrestrained Locomotion. PLoS One 8, e85321.

Towal, R. B. (2006). Right-Left Asymmetries in the Whisking Behavior of Rats Anticipate Head Movements. J. Neurosci. 26, 8838-8846.

Tuthill, J. C. and Azim, E. (2018). Proprioception. Curr. Biol. 28, R194-R203.

Tuthill, J. C. and Wilson, R. I. (2016). Mechanosensation and Adaptive Motor Control in Insects. Curr. 
Biol. 26, R1022-R1038.

816 Wang, Y., Othayoth, R. and Li, C. (2021). A robophysical model to study physical sensing of obstacles

817 in legged traversal of complex 3-D terrain. Bull. Am. Phys. Soc. 66 S14, 007.

818 Watson, J., Ritzmann, R. and Pollack, A. (2002). Control of climbing behavior in the cockroach,

819 Blaberus discoidalis . II. Motor activities associated with joint movement. J. Comp. Physiol. A

$820 \quad$ Sensory, Neural, Behav. Physiol. 188, 55-69.

821 Xuan, Q., Wang, Y. and Li, C. (2021). Environmental force sensing enables robots to traverse cluttered obstacles with interaction. IEEE Robot. Autom. Lett., in review. 